\title{
CLEFT EXTENSIONS OF HOPF ALGEBROIDS
}

\author{
GABRIELLA BÖHM AND TOMASZ BRZEZIŃSKI
}

\begin{abstract}
The notions of a cleft extension and a cross product with a Hopf algebroid are introduced and studied. In particular it is shown that an extension (with a Hopf algebroid $\left.\mathscr{H}=\left(\mathscr{H}_{L}, \mathscr{H}_{R}\right)\right)$ is cleft if and only if it is $\mathscr{H}_{R}$-Galois and has a normal basis property relative to the base ring $L$ of $\mathscr{H}_{L}$. Cleft extensions are identified as crossed products with invertible cocycles. The relationship between the equivalence classes of crossed products and gauge transformations is established. Strong connections in cleft extensions are classified and sufficient conditions are derived for the Chern-Galois characters to be independent on the choice of strong connections. The results concerning cleft extensions and crossed product are then extended to the case of weak cleft extensions of Hopf algebroids hereby defined.
\end{abstract}

\section{INTRODUCTION}

Cleft extensions of algebras by a Hopf algebra, or cleft Hopf comodule algebras, are one of the simplest and best known examples of Hopf-Galois extensions. Indeed, by [23. Theorem 9] a Hopf-Galois extension with the normal basis property is necessarily a cleft extension. With geometric interpretation of Hopf-Galois extensions over fields as non-commutative principal bundles, cleft extensions can be understood as such principal bundles that every associated bundle is trivial. Motivated by examples coming from noncommutative differential geometry, the notion of a Hopf-Galois extension was generalised to a coalgebra-Galois extension in [19], [18]. Subsequently, the notion of a cleft coalgebra extension was introduced in [15, p. 293], and most comprehensively studied in terms of cleft entwining structures in [1], [22]. The latter were extended further to weak entwining structures in [2], [3].

The aim of the present paper is to extend the theory of cleft extensions in a different direction, in the first instance motivated by recent developments in the theory of depth 2 and Frobenius ring extensions [24], [25], [26], in long term motivated by the increasing interest in Galois-type extensions with Hopf algebroid symmetries [9], [5]. Thus we introduce and study basic properties of Hopf algebroid cleft extensions. Very much as cleft extensions for Hopf algebras are an example and a testing ground for more general Hopf-Galois extensions, also Hopf algebroid cleft extensions provide one with a useful tool (or a toy model) for more general Hopf algebroid extensions. In particular, as announced in [11], cleft extensions for Hopf algebroids give a concrete illustration to the relative Chern-Galois theory. In fact the current paper can be considered as a sequel to [11] in which the ideas and results, announced in a few examples, are developed in detail and further extended. Specifically, in Section 5, sufficient conditions for the existence of (strong connection independent) relative Chern-Galois characters in Hopf algebroid cleft extensions are stated.

Date: October 2005.

1991 Mathematics Subject Classification. 16W30; 58B34; 16E40; 19 D55. 
The construction of Hopf algebroid cleft extensions, although motivated by similar ideas, is significantly different from that of cleft Hopf algebra (or coalgebra) extensions. One should remember that a Hopf algebroid involves two different coring (and bialgebroid) structures on the same $k$-module. The interplay between these intricate structures is an immanent feature of Hopf algebroid extensions. This is already present in the notion of a convolution inverse (cf. Definition 3.1), which relates two coring structures on the same $k$-module, but is perhaps most significant in the characterisation of cleft extensions in terms of the Galois and normal basis properties (cf. Theorem 3.12): a cleft $\mathscr{H}$-extension is a Galois extension with respect to the right bialgebroid $\mathscr{H}_{R}$ but it has a normal basis property with respect to the left bialgebroid $\mathscr{H}_{L}$.

In the standard Hopf algebra theory, cleft extensions of Hopf algebras are examples of crossed products with Hopf algebras: indeed a cleft extension is the same as a crossed product with an invertible cocycle (cf. [23, Theorem 11], [7, Theorem 1.18]). Motivated by this correspondence, we also develop a general theory of crossed products with bialgebroids and Hopf algebroids. In particular this involves developing the notions of a measuring and a 2-cocycle, while to relate different crossed products one needs to give meaning to gauge transformations and equivalent crossed products. In parallel to the bialgebra case, we show in Theorem 4.7 that two crossed products are equivalent if and only if one is a gauge transform of the other. We then identify cleft extensions of Hopf algebroids with crossed products with invertible cocycles (cf. Theorem 4.11 and Theorem 4.12). A generalisation of this theory to the case of weak crossed products is then outlined in Appendix.

Finally, we would like to indicate that the cleft extensions of the present paper can be placed in a broader context. A (weak) entwining structure $(A, D, \psi)$ determines a coring extension $D$ of the canonical $A$-coring $\mathscr{C}_{\psi}$ that is pure in the sense that it satisfies assumptions of [16, Theorem 2.6 (2), (arXiv version)]; see [14, Corrigendum, Definition 1]. The cleft property of an entwining structure can be formulated as a feature of $A$ as an entwined module (i.e. a $\mathscr{C}_{\psi}$-comodule). Although it is not possible to find a cleft entwining structure behind a cleft extension $B \subseteq A$ of a Hopf algebroid $\mathscr{H}$ (with left $L$-bialgebroid $\mathscr{H}_{L}$ and right $R$-bialgebroid $\mathscr{H}_{R}$ ), there is still an associated coring extension. Namely, the constituent $L$-coring in $\mathscr{H}$ is a right extension of the $A$-coring $\mathscr{C}:=A \otimes_{R} \mathscr{H}_{R}$, such that $A$ is a $\mathscr{C}$-comodule. Inspired by this observation, a unified approach to all known notions of cleft extensions in terms of pure coring extensions is developed in [14].

After publication of this paper, it turned out that the proofs of journal versions of [16, Theorem 2.6] and [9, Proposition 3.1] contain some unjustified steps. The previous (published) version of this work heavily relied on the journal version of [16. Theorem 2.6] to which we do not resort in this revision. Using informality of the arXiv, for the convenience of the readers of the earlier version, we write the corrections in blue.

Notation. Throughout this paper we work over an associative unital commutative ring $k$. An algebra means an associative unital $k$-algebra. Unit elements are denoted by 1 and multiplications by $\mu$ (or by $1_{R}, \mu_{R}$ if the algebra $R$ needs to be specified). Categories of left, right, and bimodules for an algebra $R$ are denoted by ${ }_{R} \mathbf{M}, \mathbf{M}_{R}$ and ${ }_{R} \mathbf{M}_{R}$, respectively. Their hom-sets are denoted by $\operatorname{Hom}_{R,-}(-,-) \operatorname{Hom}_{-, R}(-,-)$ and $\operatorname{Hom}_{R, R}(-,-)$, respectively.

Categories of left and right comodules for a coring $\mathscr{C}$ are denoted by ${ }^{\mathscr{C}} \mathbf{M}$ and $\mathbf{M}^{\mathscr{C}}$, respectively. For their hom-sets we write $\operatorname{Hom}^{\mathscr{C},-}(-,-)$ and $\mathrm{Hom}^{-,} \mathscr{C}(-,-)$, respectively. 


\section{PRELIMINARIES}

2.1. Bialgebroids. A bialgebroid [30],[27] can be considered as a generalisation of the notion of a bialgebra to arbitrary (non-commutative) base algebras. A (left) bialgebroid over a base algebra $L$ consists of an $L \otimes_{k} L^{o p}$-ring structure $(H, \mu, \eta)$ and an $L$-coring structure $(H, \gamma, \pi)$ on the same $k$-module $H$. Denoting the restriction of the unit map $\eta: L \otimes_{k} L^{o p} \rightarrow H$ to $L \otimes_{k} 1_{H}$ (the so called source map) by $s$ and its restriction to $1_{H} \otimes_{k} L^{o p}$ (the target map) by $t$, the bimodule structure of the $L$-coring is given by

$$
l h l^{\prime}=s(l) t\left(l^{\prime}\right) h, \quad \text { for all } l, l^{\prime} \in L, h \in H .
$$

The range of the coproduct is required to be in the Takeuchi product

$$
H \times{ }_{L} H:=\left\{\sum_{i} h_{i} \underset{L}{\otimes} h_{i}^{\prime} \in H \underset{L}{\otimes} H \mid \sum_{i} h_{i} t(l) \underset{L}{\otimes} h_{i}^{\prime}=\sum_{i} h_{i} \underset{L}{\otimes} h_{i}^{\prime} s(l) \forall l \in L\right\},
$$

which is, indeed, an algebra by factorwise multiplication. The following compatibility conditions are required between the $L \otimes_{k} L^{o p}$-ring and the $L$-coring structures.

$$
\begin{aligned}
\gamma\left(1_{H}\right) & =1_{H} \underset{L}{\otimes} 1_{H}, \\
\gamma\left(h h^{\prime}\right) & =\gamma(h) \gamma\left(h^{\prime}\right), \\
\pi\left(1_{H}\right) & =1_{L}, \\
\pi\left(h s\left(\pi\left(h^{\prime}\right)\right)\right) & =\pi\left(h h^{\prime}\right), \\
\pi\left(h t\left(\pi\left(h^{\prime}\right)\right)\right) & =\pi\left(h h^{\prime}\right),
\end{aligned}
$$

for all $l \in L$ and $h, h^{\prime} \in H$.

The $L-L$ bimodule structure of the coring, underlying a left bialgebroid, is defined in terms of the multiplication by $s(l)$ and $t(l)$ on the left. Right bialgebroids are defined analogously in terms of multiplications on the right. For more details we refer to [26].

Thus a bialgebroid is given by the following data: $k$-algebras $H$ and $L$, and maps $s$ (the source), $t$ (the target), $\gamma$ (the coproduct) and $\pi$ (the counit). We write $\mathscr{L}=(H, L, s, t, \gamma, \pi)$.

Note that if $\mathscr{L}=(H, L, s, t, \gamma, \pi)$ is a left bialgebroid then so is the co-opposite $\mathscr{L}_{\text {cop }}=$ $\left(H, L^{o p}, t, s, \gamma^{o p}, \pi\right)$, where $L^{o p}$ denotes the algebra that is isomorphic to $L$ as a $k$-module, with multiplication opposite to the one in $L$, and $\gamma^{o p}: H \rightarrow H \otimes_{L^{o p}} H, h \mapsto h_{(2)} \otimes_{L^{o p}} h_{(1)}$ is the coproduct, opposite to $\gamma: H \rightarrow H \otimes_{L} H, h \mapsto h_{(1)} \otimes_{L} h_{(2)}$. The opposite, $\mathscr{L}^{o p}=\left(H^{o p}, L\right.$, $t, s, \gamma, \pi)$ is a right bialgebroid.

2.2. Hopf algebroids. Hopf algebroids with bijective antipodes have been introduced in [13]. In [8] the definition was extended by relaxing the bijectivity of the antipode.

A Hopf algebroid consists of two (a left and a right) bialgebroid structures on the same total algebra. The source and target maps of the left bialgebroid $\mathscr{H}_{L}=\left(H, L, s_{L}, t_{L}, \gamma_{L}, \pi_{L}\right)$ and of the right bialgebroid $\mathscr{H}_{R}=\left(H, R, s_{R}, t_{R}, \gamma_{R}, \pi_{R}\right)$ are related by the following axioms.

$$
\begin{array}{lll}
s_{L} \circ \pi_{L} \circ t_{R}=t_{R}, & t_{L} \circ \pi_{L} \circ s_{R}=s_{R} & \text { and } \\
s_{R} \circ \pi_{R} \circ t_{L}=t_{L}, & & t_{R} \circ \pi_{R} \circ s_{L}=s_{L} .
\end{array}
$$

These conditions imply that the left coproduct $\gamma_{L}$ is $R-R$ bilinear and the right coproduct $\gamma_{R}$ is $L-L$ bilinear. Each coproduct is required to be left and right colinear with respect to the other bialgebroid structure, i.e. the following axioms are imposed:

$$
\left(\gamma_{L} \otimes_{R}^{\otimes} H\right) \circ \gamma_{R}=\left(H \underset{L}{\otimes} \gamma_{R}\right) \circ \gamma_{L} \quad \text { and } \quad\left(\gamma_{R} \underset{L}{\otimes} H\right) \circ \gamma_{L}=\left(H \underset{R}{\otimes} \gamma_{L}\right) \circ \gamma_{R} \text {. }
$$


An $R \otimes_{k} L-R \otimes_{k} L$ bilinear map $S: H \rightarrow H$, i.e. a $k$-linear map, such that

$$
S\left(t_{L}(l) h t_{R}(r)\right)=s_{R}(r) S(h) s_{L}(l) \quad \text { and } \quad S\left(t_{R}(r) h t_{L}(l)\right)=s_{L}(l) S(h) s_{R}(r),
$$

for $r \in R, l \in L$ and $h \in H$, is called an antipode if

$$
\mu_{H} \circ\left(S \underset{L}{\otimes_{1} H}\right) \circ \gamma_{L}=s_{R} \circ \pi_{R} \quad \text { and } \quad \mu_{H} \circ(H \underset{R}{\otimes} S) \circ \gamma_{R}=s_{L} \circ \pi_{L} .
$$

For a Hopf algebroid we use the notation $\mathscr{H}=\left(\mathscr{H}_{L}, \mathscr{H}_{R}, S\right)$.

Since in a Hopf algebroid there are two coring structures present, we use two versions of Sweedler's index notation for coproducts. For any $h \in H$, we write $\gamma_{R}(h)=h^{(1)} \otimes_{R} h^{(2)}$ (with upper indices) for the right coproduct and $\gamma_{L}(h)=h_{(1)} \otimes_{L} h_{(2)}$ (with lower indices) for the left coproduct.

Remark 2.1. In the formulation of Hopf algebroid axioms, given in (2.6), (2.7), (2.8) and (2.9), the left bialgebroid $\mathscr{H}_{L}$ and the right bialgebroid $\mathscr{H}_{R}$ play symmetric roles. It turns out, however, that this set of axioms can slightly be reduced. Namely, the second equality in (2.8) can be derived from the other axioms. This can be seen by the following computation (and its symmetric version, in which the order of multiplication and roles of $\mathscr{H}_{L}$ and $\mathscr{H}_{R}$ are interchanged). For $l \in L$ and $h \in H$,

$$
\begin{aligned}
S\left(h t_{L}(l)\right) & =S\left(t_{L}\left(\pi_{L}\left(h_{(2)}\right)\right) h_{(1)} t_{L}(l)\right)=S\left(h_{(1)} t_{L}(l)\right) s_{L}\left(\pi_{L}\left(h_{(2)}\right)\right) \\
& =S\left(h_{(1)}\right) s_{L}\left(\pi_{L}\left(h_{(2)} s_{L}(l)\right)\right)=S\left(h_{(1)}\right) h_{(2)}{ }^{(1)} s_{L}(l) S\left(h_{(2)}{ }^{(2)}\right) \\
& =S\left(h^{(1)}{ }_{(1)}\right) h^{(1)}{ }_{(2)} s_{L}(l) S\left(h^{(2)}\right)=s_{R}\left(\pi_{R}\left(h^{(1)}\right)\right) s_{L}(l) S\left(h^{(2)}\right) \\
& =s_{L}(l) s_{R}\left(\pi_{R}\left(h^{(1)}\right)\right) S\left(h^{(2)}\right)=s_{L}(l) S\left(h^{(2)} t_{R}\left(\pi_{R}\left(h^{(1)}\right)\right)\right) \\
& =s_{L}(l) S(h) .
\end{aligned}
$$

The first equality follows by the fact that $\pi_{L}$ is counit of $\gamma_{L}$ and the last one follows since $\pi_{R}$ is counit of $\gamma_{R}$. The second and the penultimate equalities follow by the first equality in (2.8). The third equality follows by the bialgebroid axiom, requiring that the range of $\gamma_{L}$ is in the Takeuchi product $H \times_{L} H$. The fourth equality follows by (2.9) and (2.6), as the latter implies - together with the left $R$-linearity of $\gamma_{R}$ - that $\gamma_{L}\left(h s_{L}(l)\right)=h_{(1)} s_{L}(l) \otimes_{L} h_{(2)}$. The fifth equality is a consequence of the right $\mathscr{H}_{R}$-colinearity of $\gamma_{L}$, i.e. (2.7). The sixth equality follows by (2.9), and the seventh one follows by (2.6), implying $s_{L}(l) s_{R}(r)=s_{R}(r) s_{L}(l)$, for $r \in R$ and $l \in L$.

It is proven in [8, Proposition 2.3] that the antipode of a Hopf algebroid is both an antimultiplicative map, i.e. $S\left(h h^{\prime}\right)=S\left(h^{\prime}\right) S(h)$, for $h, h^{\prime} \in H$, and an anti-comultiplicative map, i.e. $S(h)_{(1)} \otimes_{L} S(h)_{(2)}=S\left(h^{(2)}\right) \otimes_{L} S\left(h^{(1)}\right)$ and $S(h)^{(1)} \otimes_{R} S(h)^{(2)}=S\left(h_{(2)}\right) \otimes_{R} S\left(h_{(1)}\right)$, for $h \in H$, (note the appearance of left and right coproducts in both formulae). The maps

$$
\pi_{R} \circ t_{L}: L^{o p} \rightarrow R \quad \text { and } \quad \pi_{L} \circ s_{R}: R \rightarrow L^{o p}
$$

are inverse algebra isomorphisms.

Note that for a Hopf algebroid $\mathscr{H}=\left(\mathscr{H}_{L}, \mathscr{H}_{R}, S\right)$ also $\mathscr{H}_{c o p}^{o p}=\left(\left(\mathscr{H}_{R}\right)_{\text {cop }}^{o p},\left(\mathscr{H}_{L}\right)_{c o p}^{o p}, S\right)$ is a Hopf algebroid. If the antipode $S$ is bijective then also $\mathscr{H}_{c o p}=\left(\left(\mathscr{H}_{L}\right)_{\text {cop }},\left(\mathscr{H}_{R}\right)_{\text {cop }}, S^{-1}\right)$ and $\mathscr{H}^{o p}=\left(\left(\mathscr{H}_{R}\right)^{o p},\left(\mathscr{H}_{L}\right)^{o p}, S^{-1}\right)$ are Hopf algebroids.

Convention. Throughout, whenever it is said 'Hopf algebroid $\mathscr{H}$ ', it is meant a Hopf algebroid with all the structure modules and maps as in this section. 
2.3. Comodule algebras for bialgebroids. Let $\mathscr{R}=(H, R, s, t, \gamma, \pi)$ be a right bialgebroid and $M$ a right $\mathscr{R}$-comodule, that is, a right comodule for the $R$-coring $(H, \gamma, \pi)$. This means [20, 18.1] that $M$ is a right $R$-module and there exists a right $R$-linear coassociative and counital coaction, $\rho: M \rightarrow M \otimes_{R} H, m \mapsto m^{[0]} \otimes_{R} m^{[1]}$ (note the upper Sweedler indices indicating the involvement of a right bialgebroid). By the power of the bialgebroid structure, $M$ can be equipped with a unique left $R$-action such that $M$ becomes an $R-R$ bimodule and the range of $\rho$ is in the Takeuchi product

$$
M \times_{R} H:=\left\{\sum_{i} m_{i} \otimes_{R} h_{i} \in M \otimes_{R} H \mid \sum_{i} r m_{i} \otimes_{R} h_{i}=\sum_{i} m_{i} \otimes_{R} t(r) h_{i} \forall r \in R\right\} .
$$

The left $R$-multiplication in $M$ takes the form

$$
r m=m^{[0]} \pi\left(t(r) m^{[1]}\right) \equiv m^{[0]} \pi\left(s(r) m^{[1]}\right), \quad \text { for all } r \in R, m \in M .
$$

One checks that any $\mathscr{R}$-colinear map is $R$ - $R$ bilinear. In particular the coaction satisfies

$$
\rho\left(r m r^{\prime}\right)=m^{[0]} \otimes_{R} s(r) m^{[1]} s\left(r^{\prime}\right), \quad \text { for all } r, r^{\prime} \in R, m \in M .
$$

The category of right $\mathscr{R}$-comodules is a monoidal category with a strict monoidal functor to the category ${ }_{R} \mathbf{M}_{R}$ of $R-R$ bimodules [28, Proposition 5.6]. The $R$-action and $\mathscr{R}$-coaction on the tensor product of two comodules $M$ and $N$ are given by

$$
\left(m \otimes_{R} n\right) \cdot r=m \otimes_{R} n r, \quad\left(m \otimes_{R} n\right)^{[0]} \otimes_{R}\left(m \otimes_{R} n\right)^{[1]}=\left(m^{[0]} \otimes_{R} n^{[0]}\right) \otimes_{R} m^{[1]} n^{[1]},
$$

for all $r \in R, m \otimes_{R} n \in M \otimes_{R} N$.

A right $\mathscr{R}$-comodule algebra is a monoid in the monoidal category of right $\mathscr{R}$-comodules; hence, in particular, it is an $R$-ring.

The $R$-coring $(H, \gamma, \pi)$, underlying a right $R$-bialgebroid $\mathscr{R}$, possesses a grouplike element $1_{H}$. The coinvariants of a right $\mathscr{R}$-comodule $M$ with respect to the grouplike element $1_{H}$ are the elements of

$$
M^{c o \mathscr{R}}=\left\{m \in M \mid m^{[0]} \otimes_{R} m^{[1]}=m \otimes_{R} 1_{H}\right\} .
$$

It is straightforward to check that if $A$ is a right $\mathscr{R}$-comodule algebra, then its coinvariants form a subalgebra $B:=A^{c o \mathscr{R}}$. In this case the algebra extension $B \subseteq A$ is termed a right $\mathscr{R}$-extension.

A right $\mathscr{R}$-extension $B \subseteq A$ is a right $\mathscr{R}$-Galois extension if the canonical map

$$
\operatorname{can}_{R}: A \otimes_{B} A \rightarrow A \otimes_{R} H, \quad a \otimes_{B} a^{\prime} \mapsto a a^{\prime[0]} \bigotimes_{R} a^{\prime[1]},
$$

is bijective, i.e. the $A$-coring $A \otimes_{R} H$ with the coproduct $A \otimes_{R} \gamma$, the counit $A \otimes_{R} \pi$ and with the $A$ - $A$ bimodule structure $a_{1}\left(a \otimes_{R} h\right) a_{2}=a_{1} a a_{2}{ }^{[0]} \otimes_{R} h a_{2}{ }^{[1]}$, is a Galois coring [24].

Analogously, a right comodule $N$, with coaction $n \mapsto n_{[0]} \otimes_{L} n_{[1]}$ (note the lower Sweedler indices indicating the involvement of a left bialgebroid), for the $L$-coring $(H, \gamma, \pi)$, underlying a left bialgebroid $\mathscr{L}=(H, L, s, t, \gamma, \pi)$, can be equipped with a left $L$-action

$$
\ln =n_{[0]} \pi\left(n_{[1]} s(l)\right) \equiv n_{[0]} \pi\left(n_{[1]} t(l)\right), \quad \text { for all } l \in L, n \in N .
$$

The category of right $\mathscr{L}$-comodules is a monoidal category with monoidal product, the module tensor product over $L^{o p}$. The right $L$-action and $\mathscr{L}$-coaction on the tensor product of two $\mathscr{L}$-comodules $M$ and $N$ are

$$
\left(m_{L^{o p}}^{\otimes} n\right) \cdot l=m l_{L^{o p}}^{\otimes} n, \quad\left(m_{L^{o p}}^{\otimes} n\right)_{[0]} \underset{L}{\otimes}\left(m_{L^{o p}}^{\otimes} n\right)_{[1]}=\left(m_{[0]} \otimes_{L^{o p}} n_{[0]}\right) \otimes_{L}^{\otimes} m_{[1]} n_{[1]},
$$


for all $l \in L, m \otimes_{L^{o p}} n \in M \otimes_{L^{o p}} N$. Right $\mathscr{L}$-comodule algebras are defined as monoids in the monoidal category of right $\mathscr{L}$-comodules - hence they are, in particular, $L^{o p}$-rings. Coinvariants are defined with respect to the grouplike element $1_{H}$. An algebra extension $B \subseteq A$ is called a right $\mathscr{L}$-extension if $A$ is a right $\mathscr{L}$-comodule algebra and $B=A^{\text {co } \mathscr{L}}$. A right $\mathscr{L}$-extension $B \subseteq A$ is said to be right $\mathscr{L}$-Galois if the canonical map

$$
\operatorname{can}_{L}: A \underset{B}{\otimes} A \rightarrow A \underset{L}{\otimes} H, \quad a \otimes_{B} a^{\prime} \mapsto a_{[0]} a^{\prime} \otimes_{L} a_{[1]},
$$

is bijective.

Right comodules for a left bialgebroid $\mathscr{L}$ are canonically identified with left comodules

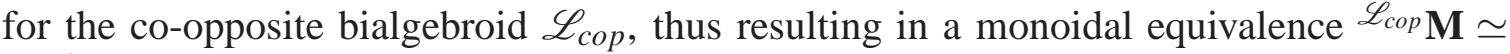
$\mathbf{M}^{\mathscr{L}}$. This identification leads to analogous notions of left comodule algebras, left $\mathscr{L}$ extensions and left $\mathscr{L}$-Galois extensions.

2.4. Comodule algebras for Hopf algebroids. In a Hopf algebroid $\mathscr{H}$ there are two bialgebroid, hence two coring structures present. The definition of an $\mathscr{H}$-comodule in [9, Definition 3.2] and [5, Section 2.2] refers to them in a symmetrical way:

Definition 2.2. A right comodule of a Hopf algebroid $\mathscr{H}$ is a right $L$-module as well as a right $R$-module $M$, together with a right coaction $\rho_{R}: M \rightarrow M \otimes_{R} H$ of the constituent right bialgebroid $\mathscr{H}_{R}$ and a right coaction $\rho_{L}: M \rightarrow M \otimes_{L} H$ of the constituent left bialgebroid $\mathscr{H}_{L}$, such that $\rho_{R}$ is an $\mathscr{H}_{L}$-comodule map and $\rho_{L}$ is an $\mathscr{H}_{R}$-comodule map. Explicitly,

$$
\left(M \otimes_{R} \gamma_{L}\right) \circ \rho_{R}=\left(\rho_{R} \otimes_{L} H\right) \circ \rho_{L} \quad \text { and } \quad\left(M \otimes_{L} \gamma_{R}\right) \circ \rho_{L}=\left(\rho_{L} \otimes_{R} H\right) \circ \rho_{R} .
$$

Morphisms of $\mathscr{H}$-comodules are $\mathscr{H}_{R}$-comodule maps as well as $\mathscr{H}_{L}$-comodule maps. The category of right $\mathscr{H}$-comodules is denoted by $\mathbf{M}^{\mathscr{H}}$.

The category ${ }^{\mathscr{H}} \mathbf{M}$ of left $\mathscr{H}$-comodules is defined symmetrically.

Note that since the right $R$ - and $L$-actions on $H$ commute, also any right $\mathscr{H}$-comodule is a right $R \otimes_{k} L$-module.

Remark 2.3. The antipode $S$ in a Hopf algebroid $\mathscr{H}$ defines a functor ${ }^{\mathscr{H}} \mathbf{M} \rightarrow \mathbf{M}^{\mathscr{H}}$. Indeed, if $M$ is a left $\mathscr{H}$-comodule, with $\mathscr{H}_{R}$-coaction $m \mapsto m^{[-1]} \otimes_{R} m^{[0]}$ and $\mathscr{H}_{L}$-coaction $m \mapsto$ $m_{[-1]} \otimes_{L} m_{[0]}$, then it is a right $\mathscr{H}$-comodule with right $R$-action $m r:=\pi_{L}\left(t_{R}(r)\right) m$, right $L$-action $m l:=\pi_{R}\left(t_{L}(l)\right) m$ and respective coactions

$$
m \mapsto m_{[0]} \otimes_{R} S\left(m_{[-1]}\right) \quad \text { and } \quad m \mapsto m^{[0]} \otimes_{L} S\left(m^{[-1]}\right) .
$$

Left $\mathscr{H}$-comodule maps are also right $\mathscr{H}$-comodule maps for these coactions.

A functor $\mathbf{M}^{\mathscr{H}} \rightarrow{ }^{\mathscr{H}} \mathbf{M}$ is constructed symmetrically.

Proposition 2.4. Let $\mathscr{H}$ be a Hopf algebroid and $\left(M, \rho_{L}, \rho_{R}\right)$ be a right $\mathscr{H}$-comodule. Then any coinvariant of the $\mathscr{H}_{R}$-comodule $\left(M, \rho_{R}\right)$ is coinvariant also for the $\mathscr{H}_{L}$-comodule $\left(M, \rho_{L}\right)$.

If moreover the antipode of $\mathscr{H}$ is bijective then coinvariants of the $\mathscr{H}_{R}$-comodule $\left(M, \rho_{R}\right)$ and the $\mathscr{H}_{L}$-comodule $\left(M, \rho_{L}\right)$ coincide.

Proof. For a right $\mathscr{H}$-comodule $\left(M, \rho_{L}, \rho_{R}\right)$, consider the map

$$
\Phi_{M}: M \otimes_{R} H \rightarrow M \otimes_{L} H, \quad m \otimes_{R} h \mapsto \rho_{L}(m) S(h),
$$

where $H$ is a left $L$-module via the source map $s_{L}$ and a left $R$-module via the target map $t_{R}$, and $M \otimes_{L} H$ is understood to be a right $H$-module via the second factor. Since $\Phi_{M}\left(\rho_{R}(m)\right)=$ 
$m \otimes_{L} 1_{H}$ and $\Phi_{M}\left(m \otimes_{R} 1_{H}\right)=\rho_{L}(m)$, we have the first claim in Proposition 2.4 proven. In order to prove the second assertion, note that if $S$ is an isomorphism then so is $\Phi_{M}$, with the inverse $\Phi_{M}^{-1}\left(m \otimes_{L} h\right)=S^{-1}(h) \rho_{R}(m)$, where $M \otimes_{R} H$ is understood to be a left $H$-module via the second factor.

Although the functors $U$ and $V$ in Theorem 2.5 below are not known to exist without further assumptions, they exist in all known examples of Hopf algebroids and they establish isomorphisms between the categories of $\mathscr{H}_{L}$-comodules, $\mathscr{H}_{R}$-comodules and $\mathscr{H}$ comodules. In the following theorem $F_{R}$ and $F_{L}$ denote the forgetful functors $\mathbf{M}^{\mathscr{H}_{R}} \rightarrow \mathbf{M}_{k}$ and $\mathbf{M}^{\mathscr{H}_{L}} \rightarrow \mathbf{M}_{k}$, respectively, while $G_{R}$ and $G_{L}$ denote the forgetful functors $\mathbf{M}^{\mathscr{H}} \rightarrow \mathbf{M}^{\mathscr{H}_{R}}$ and $\mathbf{M}^{\mathscr{H}} \rightarrow \mathbf{M}^{\mathscr{H}_{L}}$, respectively. $H$ is regarded as an $R$-bimodule via right multiplication by $s_{R}$ and $t_{R}$ and an $L$-bimodule via left multiplication by $s_{L}$ and $t_{L}$.

Theorem 2.5. Consider a Hopf algebroid $\mathscr{H}$.

(1) If the equaliser

$$
M \stackrel{\rho_{R}}{\longrightarrow} M \otimes_{R} H \underset{M \otimes_{R} \gamma_{R}}{\stackrel{\rho_{R} \otimes_{R} H}{\longrightarrow}} M \otimes_{R} H \otimes_{R} H
$$

in $\mathbf{M}_{L}$ is $H \otimes_{L} H$-pure, i.e. it is preserved by the functor $(-) \otimes_{L} H \otimes_{L} H: \mathbf{M}_{L} \rightarrow \mathbf{M}_{L}$, for any right $\mathscr{H}_{R}$-comodule $\left(M, \rho_{R}\right)$, then there exists a functor $U: \mathbf{M}^{\mathscr{H}_{R}} \rightarrow \mathbf{M}^{\mathscr{H}_{L}}$, such that $F_{L} \circ U=F_{R}$ and $U \circ G_{R}=G_{L}$. In particular, $G_{R}$ is full.

(2) If the equaliser

$$
N \stackrel{\rho_{L}}{\longrightarrow} N \otimes_{L} H \underset{N \otimes_{L} \gamma_{L}}{\stackrel{\rho_{L} \otimes_{L} H}{\longrightarrow}} N \otimes_{L} H \otimes_{L} H
$$

in $\mathbf{M}_{R}$ is $H \otimes_{R} H$-pure, i.e. it is preserved by the functor $(-) \otimes_{R} H \otimes_{R} H: \mathbf{M}_{R} \rightarrow \mathbf{M}_{R}$, for any right $\mathscr{H}_{L}$-comodule $\left(N, \rho_{L}\right)$, then there exists a functor $V: \mathbf{M}^{\mathscr{H}_{L}} \rightarrow \mathbf{M}^{\mathscr{H}_{R}}$, such that $F_{R} \circ V=F_{L}$ and $V \circ G_{L}=G_{R}$. In particular, $G_{L}$ is full.

(3) If both purity assumptions in parts (1) and (2) hold, then the forgetful functors $G_{R}: \mathbf{M}^{\mathscr{H}} \rightarrow \mathbf{M}^{\mathscr{H} R}$ and $G_{L}: \mathbf{M}^{\mathscr{H}} \rightarrow \mathbf{M}^{\mathscr{H}_{L}}$ are isomorphisms, hence $U$ and $V$ are inverse isomorphisms.

Proof. (1) Recall that (2.22) defines the $\mathscr{H}_{R}$-cotensor product $M \square_{\mathscr{H}_{R}} H \simeq M$. By (2.7), $H$ is an $\mathscr{H}_{R}-\mathscr{H}_{L}$ bicomodule, with left coaction $\gamma_{R}$ and right coaction $\gamma_{L}$. Thus in light of [20, 22.3] and its Erratum, we can define a desired functor $U:=(-) \square_{\mathscr{H}_{R}} H$. Clearly, it satisfies $F_{L} \circ U=F_{R}$. For an $\mathscr{H}$-comodule $\left(M, \rho_{L}, \rho_{R}\right)$, the coaction on the $\mathscr{H}_{L}$-comodule $U\left(G_{R}\left(M, \rho_{L}, \rho_{R}\right)\right)=U\left(M, \rho_{R}\right)$ is given by

$$
M \stackrel{\rho_{R}}{\longrightarrow} M \square_{\mathscr{H}_{R}} H \stackrel{M \square \mathscr{H}_{R} \gamma_{L}}{\longrightarrow} M \square_{\mathscr{H}_{R}}\left(H \otimes_{L} H\right) \stackrel{\simeq}{\longrightarrow}\left(M \square_{\mathscr{H}_{R}} H\right) \otimes_{L} H \stackrel{M \otimes_{R} \pi_{R} \otimes_{L} H}{\longrightarrow} M \otimes_{L} H,
$$

where in the third step we used that since the equaliser (2.22) is $H \otimes_{L} H$-pure, it is in particular $H$-pure. Using that $\rho_{R}$ is a right $\mathscr{H}_{L}$-comodule map and counitality of $\rho_{R}$, we conclude that (2.23) is equal to $\rho_{L}$. Hence $U \circ G_{R}=G_{L}$. This proves that for any two $\mathscr{H}$-comodules $M$ and $M^{\prime}$, and any $\mathscr{H}_{R}$-comodule map $f: M \rightarrow M^{\prime}, U(f)=f$ is an $\mathscr{H}_{L}$-comodule map hence an $\mathscr{H}$-comodule map, i.e. that $G_{R}$ is full. Part (2) is proven symmetrically.

(3) For the functor $U$ in part (1) and a right $\mathscr{H}_{R}$-comodule $\left(M, \rho_{R}\right)$, denote $U\left(M, \rho_{R}\right)=$ : $\left(M, \rho_{L}\right)$. With this notation, define a functor $\widehat{G}_{R}: \mathbf{M}^{\mathscr{H}} \mathcal{H}_{R} \rightarrow \mathbf{M}^{\mathscr{H}}$, with object map $\left(M, \rho_{R}\right) \mapsto$ $\left(M, \rho_{L}, \rho_{R}\right)$, and acting on the morphisms as the identity map. Being coassociative, $\rho_{R}$ is 
an $\mathscr{H}_{R}$-comodule map, so by part (1) it is an $\mathscr{H}_{L}$-comodule map. Symmetrically, by part (2) $\rho_{L}$ is an $\mathscr{H}_{R}$-comodule map. So $\widehat{G}_{R}$ is a well defined functor. We claim that it is the inverse of $G_{R}$. Obviously, $G_{R} \circ \widehat{G}_{R}$ is the identity functor. In the opposite order, note that by construction $G_{L} \circ \widehat{G}_{R}=U$. Therefore, $G_{L} \circ \widehat{G}_{R} \circ G_{R}=U \circ G_{R}=G_{L}$, cf. part (1). That is, $\widehat{G}_{R} \circ G_{R}$ takes an $\mathscr{H}$-comodule $\left(M, \rho_{L}, \rho_{R}\right)$ to the same $\mathscr{H}_{L}$-comodule $\left(M, \rho_{L}\right)$. Since $\widehat{G}_{R} \circ G_{R}$ obviously takes $\left(M, \rho_{L}, \rho_{R}\right)$ to the same $\mathscr{H}_{R}$-comodule $\left(M, \rho_{R}\right)$ as well, we conclude that also $\widehat{G}_{R} \circ G_{R}$ is the identity functor.

In a symmetrical way, in terms of the functor $V\left(N, \rho_{L}\right)=:\left(N, \rho_{R}\right)$ in part (2), one constructs $G_{L}^{-1}$ with object map $\left(N, \rho_{L}\right) \mapsto\left(N, \rho_{L}, \rho_{R}\right)$, and acting on the morphisms as the identity map. The identities $G_{L} \circ G_{R}^{-1}=U$ and $G_{R} \circ G_{L}^{-1}=V$ prove that $U$ and $V$ are mutually inverse isomorphisms, as stated.

Example 2.6. We list some families of Hopf algebroids $\mathscr{H}$ in which the purity (i.e. equaliserpreserving) conditions in Theorem 2.5 hold.

(1) All purity conditions in Theorem 2.5 hold if $H$ is flat as a left $L$ - and a left $R$-module. Indeed, in this case the functors $(-) \otimes_{L} H: \mathbf{M}_{L} \rightarrow \mathbf{M}_{L}$ and $(-) \otimes_{R} H: \mathbf{M}_{R} \rightarrow \mathbf{M}_{R}$ preserve any equaliser. In particular, Frobenius Hopf algebroids in [5] (being finitely generated and projective) are flat.

(2) Weak Hopf algebras, introduced in [12], determine Hopf algebroids over Frobeniusseparable base algebras $L \simeq R^{o p}$, cf. [10, 4.1.2]. Recall that Frobenius separability of a $k$-algebra $R$ means the existence of a $k$-module map $\psi: R \rightarrow k$ and an element $\sum_{i} e_{i} \otimes_{k} f_{i} \in$ $R \otimes_{k} R$, such that

$$
\sum_{i} \psi\left(r e_{i}\right) f_{i}=r=\sum_{i} e_{i} \psi\left(f_{i} r\right), \quad \text { for all } r \in R, \quad \text { and } \quad \sum_{i} e_{i} f_{i}=1_{R} .
$$

Note that this implies that $\sum_{i} r e_{i} \otimes_{k} f_{i}=\sum_{i} e_{i} \otimes_{k} f_{i} r$, for all $r \in R$ (hence the name separable). For a Frobenius-separable algebra $R$, any right $R$-module $X$ and left $R$-module $Y$, the canonical epimorphism $X \otimes_{k} Y \rightarrow X \otimes_{R} Y$ is split by $x \otimes_{R} y \mapsto \sum_{i} x e_{i} \otimes_{k} f_{i} y$. For the base algebras of a weak Hopf algebra, the Frobenius-separability structure arises from the restriction of the counit and the image of the unit element $1_{H}$ under the coproduct.

Let $H$ be a weak Hopf algebra with (weak) coproduct $\Delta: H \rightarrow H \otimes_{k} H$. Denote its left and right (or 'target' and 'source') subalgebras by $L$ and $R$, respectively. (These serve as the base algebras of the corresponding Hopf algebroid, see [10].) Note that $\Delta$ is an $R-L$ bimodule map.

Any right comodule $(M, \rho)$ of a weak Hopf algebra $H$ (as a coalgebra) can be equipped with a right $R$-action via $m r=m_{<0>} \varepsilon\left(m_{<1>} r\right)$, where $\rho(m)=m_{<0>} \otimes_{k} m_{<1>}$ and $\varepsilon$ denotes the counit of $H$. Moreover, any right comodule $(M, \rho)$ of $H$ yields a right coaction of the constituent right bialgebroid, by composing $\rho$ with the (split) epimorphism $p_{M}: M \otimes_{k} H \rightarrow$ $M \otimes_{R} H$. Define a right $L$-module structure on $M$ via (2.22).

For any left $L$-module $N$, consider the following diagram (in $\mathbf{M}_{k}$ ).

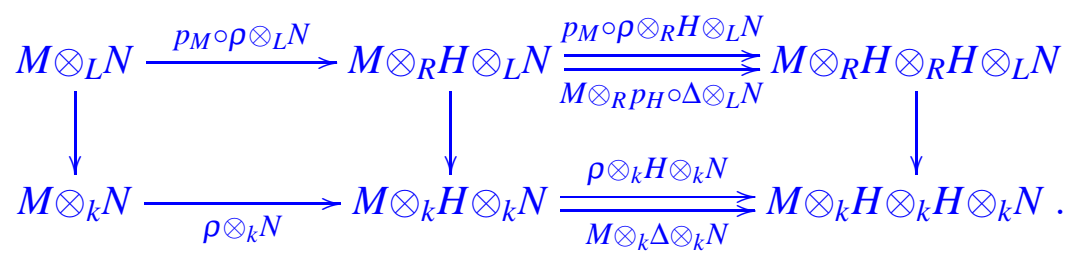


The $R$-actions on $H$ are given by right multiplications by the source and target maps and the $L$-actions on $H$ are given by left multiplications. The vertical arrows denote the sections of the canonical epimorphisms, given by the Frobenius-separability structures of $L$ and $R$. The diagram is easily checked to be serially commutative (meaning commutativity with either simultaneous choice of the upper or the lower ones of the parallel arrows). Clearly,

$$
M \stackrel{\rho}{\longrightarrow} M \otimes_{k} H \underset{M \otimes_{k} \Delta}{\stackrel{\rho \otimes_{k} H}{\longrightarrow}} M \otimes_{k} H \otimes_{k} H
$$

is a split equaliser in $\mathbf{M}_{k}$ (with splitting provided by the counit of $H$ ), hence the bottom row of the diagram in (2.24) is an equaliser. This implies that also the top row is an equaliser, so in particular the purity conditions in Theorem 2.5 (1) hold. The conditions in Theorem 2.5 (2) are verified by a symmetrical reasoning.

(3) For any $k$-algebra $L$, the tensor product algebra $H:=L \otimes_{k} L^{o p}$ carries a Hopf algebroid structure, see [10,4.1.3]. Since the left $L$-action on $H$ is given by multiplication in the first factor, the functors $F\left((-) \otimes_{L} H \otimes_{L} H\right)$ and $F(-) \otimes_{k} L \otimes_{k} L: \mathbf{M}_{L} \rightarrow \mathbf{M}_{k}$ are naturally isomorphic, where $F: \mathbf{M}_{L} \rightarrow \mathbf{M}_{k}$ denotes the forgetful functor. The forgetful functor $F$ has a left adjoint, hence it preserves any equaliser. The functor $F$ takes (2.22) to a split equaliser (with splitting provided by $\pi_{R}$ ), which is then preserved by any functor. This proves that $F(-) \otimes_{k} L \otimes_{k} L$ and hence $F\left((-) \otimes_{L} H \otimes_{L} H\right)$ preserve (2.22). Since $F$ also reflects equalisers, we conclude that (2.22) is preserved by $(-) \otimes_{L} H \otimes_{L} H: \mathbf{M}_{L} \rightarrow \mathbf{M}_{L}$. The purity conditions in Theorem 2.5 (2) are proven to hold similarly.

(4) In [4, Corrigendum], the purity conditions in Theorem 2.5 are proven to hold for a Hopf algebroid whose constituent $R$-coring (equivalently, the constituent $L$-coring) is coseparable.

Theorem 2.7. For any Hopf algebroid $\mathscr{H}, \mathbf{M}^{\mathscr{H}}$ is a monoidal category. Moreover, there are strict monoidal forgetful functors rendering commutative the following diagram:

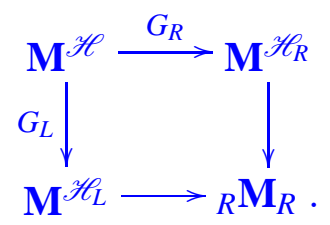

Proof. Commutativity of the diagram follows by comparing the unique $R$-actions that make $R$-bilinear the $\mathscr{H}_{R}$-coaction and the $\mathscr{H}_{L}$-coaction in an $\mathscr{H}$-comodule, respectively, (see (2.12) and (2.16), and the algebra isomorphism (2.10). Strict monoidality of the functors on the right hand side and in the bottom row follows by [28, Theorem 5.6] (and its application to the opposite of the bialgebroid $\mathscr{H}_{L}$ ), cf. Section 2.3 . In order to see strict monoidality of the remaining two functors $G_{R}$ and $G_{L}$, recall that by [28, Theorem 5.6] (applied to $\mathscr{H}_{R}$ and the opposite of $\mathscr{H}_{L}$ ), the $R$-module tensor product of any two $\mathscr{H}$ comodules is an $\mathscr{H}_{R}$-comodule and an $\mathscr{H}_{L}$-comodule, via the diagonal coactions, cf. (2.14) and (2.17). It is straightforward to check compatibility of these coactions in the sense of Definition 2.2. Similarly, $R\left(\simeq L^{o p}\right)$ is known to be an $\mathscr{H}_{R}$-comodule and an $\mathscr{H}_{L}$-comodule, and compatibility of the coactions is obvious. Finally, the $R$-module tensor product of $\mathscr{H}$ comodule maps is an $\mathscr{H}_{R}$-comodule map and an $\mathscr{H}_{L}$-comodule map by [28, Theorem 5.6]. Thus it is an $\mathscr{H}$-comodule map. By Theorem [28, Theorem 5.6] also the coherence natural transformations in ${ }_{R} \mathbf{M}_{R}$ are $\mathscr{H}_{R^{-}}$and $\mathscr{H}_{L}$-comodule maps, so $\mathscr{H}$-comodule maps, what completes the proof. 
Definition 2.8. A right comodule algebra of a Hopf algebroid $\mathscr{H}$ is a monoid in the monoidal category $\mathbf{M}^{\mathscr{H}}$ of right $\mathscr{H}$-comodules. Explicitly, an $R$-ring $(A, \mu, \eta)$, such that $A$ is a right $\mathscr{H}$-comodule and $\eta: R \rightarrow A$ and $\mu: A \otimes_{R} A \rightarrow A$ are right $\mathscr{H}$-comodule maps. Using the notations $a \mapsto a^{[0]} \otimes_{R} a^{[1]}$ and $a \mapsto a_{[0]} \otimes_{L} a_{[1]}$ for the $\mathscr{H}_{R^{-}}$and $\mathscr{H}_{L^{-} \text {-coactions, re- }}$ spectively, $\mathscr{H}$-colinearity of $\eta$ and $\mu$ means the identities, for all $a, a^{\prime} \in A$,

$$
\begin{aligned}
& 1_{A}{ }^{[0]} \otimes_{R} 1_{A}{ }^{[1]}=1_{A} \otimes_{R} 1_{H}, \quad\left(a a^{\prime}\right)^{[0]} \otimes_{R}\left(a a^{\prime}\right)^{[1]}=a^{[0]} a^{\prime[0]} \otimes_{R} a^{[1]} a^{\prime[1]} \\
& 1_{A[0]} \otimes_{L} 1_{A[1]}=1_{A} \otimes_{L} 1_{H}, \quad\left(a a^{\prime}\right)_{[0]} \otimes_{L}\left(a a^{\prime}\right)_{[1]}=a_{[0]} a_{[0]}^{\prime} \otimes_{L} a_{[1]} a_{[1]}^{\prime} \text {. }
\end{aligned}
$$

Symmetrically, a left $\mathscr{H}$-comodule algebra is a monoid in ${ }^{\mathscr{H}} \mathbf{M}$.

If $A$ is a right comodule algebra of a Hopf algebroid $\mathscr{H}$, with $\mathscr{H}_{R}$-coinvariant subalgebra $B$, then we say that $B \subseteq A$ is a (right) $\mathscr{H}$-extension.

The functors in Remark 2.3 induced by the antipode are checked to be strictly antimonoidal. Therefore, the opposite of a right $\mathscr{H}$-comodule algebra, with coactions in Remark 2.3, is a left $\mathscr{H}$-comodule algebra and conversely.

Whenever the antipode in a Hopf algebroid $\mathscr{H}$ is bijective, it induces strict anti-monoidal isomorphisms ${ }^{\mathscr{H}} \mathbf{M} \rightarrow \mathbf{M}^{\mathscr{H}} \simeq \mathscr{H}_{c o p} \mathbf{M}$ and $\mathbf{M}^{\mathscr{H}} \rightarrow{ }^{\mathscr{H}} \mathbf{M} \simeq \mathbf{M}^{\mathscr{H}_{c o p}}$.

\section{3. $\mathscr{H}$-CLEFT EXTENSIONS}

Recall that to an $L$-ring $A$ (with multiplication $\mu: A \otimes_{L} A \rightarrow A$ and unit map $\eta: L \rightarrow A$ ) and an $L$-coring $H$ (with comultiplication $\gamma: H \rightarrow H \otimes_{L} H$ and counit $\pi: H \rightarrow L$ ), one associates a convolution algebra $\operatorname{Hom}_{L, L}(H, A)$, with multiplication $j \diamond j^{\prime}:=\mu \circ\left(j \otimes_{L} j^{\prime}\right) \circ \gamma$ and unit $\eta \circ \pi$. The first aim of this section is to develop a generalisation of the notion of a convolution algebra and, in particular, of a convolution inverse suitable for Hopf algebroids. This will make it possible to interpret in particular the antipode of a Hopf algebroid as the convolution inverse of the identity map.

As explained in Section 2.2, a Hopf algebroid is built on a $k$-module with two coring structures. Although we are primarily interested in Hopf algebroids, in general there is no need to put any special restrictions on these coring structures. Dually, one can consider a $k$-module with ring structures over two different rings. In this more general situation the convolution algebra (which is simply a $k$-linear category with a single object) can be generalised to a Morita context (i.e. a $k$-linear category with two objects). The notion of a convolution inverse is introduced within this convolution category.

Let $L$ and $R$ be $k$-algebras and let $H$ and $A$ be $k$-modules. Assume that $A$ is an $L$-ring (with multiplication $\mu_{L}: A \otimes_{L} A \rightarrow A$ and unit $\eta_{L}: L \rightarrow A$ ) and an $R$-ring (with multiplication $\mu_{R}: A \otimes_{R} A \rightarrow A$ and unit $\eta_{R}: R \rightarrow A$ ). Assume that $A$ is an $L-R$ and $R$ - $L$ bimodule with respect to the corresponding module structures, $\mu_{L}$ is $R-R$ bilinear and $\mu_{R}$ is $L-L$ bilinear, and that

$$
\mu_{L} \circ\left(A \underset{L}{\otimes} \mu_{R}\right)=\mu_{R} \circ\left(\mu_{L} \underset{R}{\otimes} A\right), \quad \mu_{R} \circ\left(A \underset{R}{\otimes} \mu_{L}\right)=\mu_{L} \circ\left(\mu_{R} \otimes_{L} A\right) .
$$

Dually, assume that $H$ is an $L$-coring (with comultiplication $\gamma_{L}: H \rightarrow H \otimes_{L} H$ and counit $\pi_{L}: H \rightarrow L$ ) and an $R$-coring (with comultiplication $\gamma_{R}: H \rightarrow H \otimes_{R} H$ and counit $\pi_{R}: H \rightarrow$ $R$ ). Assume further that $H$ is an $L-R$ and $R-L$ bimodule with respect to the corresponding module structures, such that $\gamma_{L}$ is $R-R$ bilinear, $\gamma_{R}$ is $L-L$ bilinear and

$$
\left(H \underset{L}{\otimes} \gamma_{R}\right) \circ \gamma_{L}=\left(\gamma_{L} \underset{R}{\otimes} H\right) \circ \gamma_{R}, \quad\left(H \underset{R}{\otimes} \gamma_{L}\right) \circ \gamma_{R}=\left(\gamma_{R} \otimes_{L} H\right) \circ \gamma_{L} .
$$


To the above data one associates a $k$-linear convolution category $\operatorname{Conv}(H, A)$ as follows. Conv $(H, A)$ has two objects, $R$ and $L$, and morphisms

$$
\operatorname{Conv}(H, A)(P, Q)=\operatorname{Hom}_{Q, P}(H, A), \quad P, Q \in\{L, R\},
$$

with composition $\diamond$, defined for all $\phi \in \operatorname{Hom}_{P, Q}(H, A)$ and $\psi \in \operatorname{Hom}_{Q, S}(H, A), P, Q, S \in$ $\{L, R\}$,

$$
\phi \diamond \psi=\mu_{Q} \circ\left(\phi_{Q}^{\otimes} \psi\right) \circ \gamma_{Q} \in \operatorname{Hom}_{P, S}(H, A) .
$$

Note that the identity morphism in $\operatorname{Conv}(H, A)(P, P)$ is $\eta_{P} \circ \pi_{P}$. The conditions (3.1) and (3.2) together with coassociativity of the coproducts in $H$ and associativity of products in $A$ ensure that the composition $\diamond$ is an associative operation.

Definition 3.1. Let $\operatorname{Conv}(H, A)$ be a convolution category and let $j$ be a morphism in $\operatorname{Conv}(H, A)$. A retraction of $j$ in $\operatorname{Conv}(H, A)$ is called a left convolution inverse of $j$ and a section of $j$ in $\operatorname{Conv}(H, A)$ is called a right convolution inverse of $j$. If $j$ is an isomorphism in $\operatorname{Conv}(H, A)$, then it is said to be convolution invertible; its inverse is called the convolution inverse of $j$ and is denoted by $j^{c}$.

Remark 3.2. (1) A $k$-linear category with a single object $a$ can be identified with the $k$ algebra $\operatorname{End}(a)$ of the morphisms in the category. In a similar manner, a $k$-linear category with two objects $a$ and $b$ can be identified with a Morita context as follows. The composition of morphisms makes $k$-modules $\operatorname{Hom}(a, b)$ and $\operatorname{Hom}(b, a)$ bimodules for $k$-algebras $\operatorname{End}(a)$ and $\operatorname{End}(b)$. Furthermore, the restriction of the composition to the map

$$
\operatorname{Hom}(a, b) \underset{k}{\otimes} \operatorname{Hom}(b, a) \rightarrow \operatorname{End}(b)
$$

is an $\operatorname{End}(a)$-balanced $\operatorname{End}(b)$-bimodule map. That is, it is a composite of the canonical epimorphism $\operatorname{Hom}(a, b) \otimes_{k} \operatorname{Hom}(b, a) \rightarrow \operatorname{Hom}(a, b) \otimes_{\operatorname{End}(a)} \operatorname{Hom}(b, a)$, and an $\operatorname{End}(b)-$ bimodule map, $F_{a}: \operatorname{Hom}(a, b) \otimes_{\operatorname{End}(a)} \operatorname{Hom}(b, a) \rightarrow \operatorname{End}(b)$. Similarly, the map

$$
\operatorname{Hom}(b, a) \underset{k}{\otimes} \operatorname{Hom}(a, b) \rightarrow \operatorname{End}(a),
$$

obtained by restricting the composition, factors through the canonical epimorphism and the $\operatorname{End}(a)$-bimodule map, $F_{b}: \operatorname{Hom}(b, a) \otimes_{\operatorname{End}(b)} \operatorname{Hom}(a, b) \rightarrow \operatorname{End}(a)$. Using the associativity of the composition of the morphisms in a category, one easily checks that the 6-tuple $\left(\operatorname{End}(a), \operatorname{End}(b), \operatorname{Hom}(b, a), \operatorname{Hom}(a, b), F_{a}, F_{b}\right)$ is a Morita context. Clearly, there is a category of this kind behind any Morita context.

In particular, the convolution category $\operatorname{Conv}(H, A)$ can be identified with a Morita context connecting convolution algebras $\operatorname{Hom}_{L, L}(H, A)$ and $\operatorname{Hom}_{R, R}(H, A)$.

(2) In the case $L=R, \gamma_{L}=\gamma_{R}, \pi_{L}=\pi_{R}, \mu_{R}=\mu_{L}, \eta_{R}=\eta_{L}$, i.e. when there is one, say, $L$-coring $H$ and one, say, $L$-ring $A$, the convolution category Conv $(H, A)$ consists of a single object. The algebras in the corresponding Morita context are both equal to the convolution algebra $\operatorname{Hom}_{L, L}(H, A)$, the bimodules are the regular bimodules and the connecting homomorphisms are both equal to the identity map of $\operatorname{Hom}_{L, L}(H, A)$. In a word: the Morita context reduces to the convolution algebra. Thus an $L-L$ bimodule map $j$ is convolution invertible in the sense of Definition 3.1 if and only if it is an invertible element of the convolution algebra $\operatorname{Hom}_{L, L}(H, A)$.

(3) Conditions (3.1), imposed on the $R$-ring and $L$-ring structures of $A$, imply that the underlying $k$-algebras are isomorphic via the map $A \ni a \mapsto \mu_{R}\left(a \otimes_{R} \eta_{L}\left(1_{L}\right)\right)$, with the inverse $a \mapsto \mu_{L}\left(a \otimes_{L} \eta_{R}\left(1_{R}\right)\right)$. 
(4) Conditions (3.2), imposed on the two coring structures of $H$, imply that the $L$-coring $H$ is a left (and right) extension of the $R$-coring $H$, while the $R$-coring $H$ is a right (and left) extension of the $L$-coring $H$ in the sense of [16], with the coactions given by the coproducts.

We can now exemplify the contents of Definition 3.1 with the main case of interest, whereby the coring structures on $H$ constitute bialgebroids. Consider a right bialgebroid $\mathscr{H}_{R}=\left(H, R, s_{R}, t_{R}, \gamma_{R}, \pi_{R}\right)$ and a left bialgebroid $\mathscr{H}_{L}=\left(H, L, s_{L}, t_{L}, \gamma_{L}, \pi_{L}\right)$ on the same total algebra $H$, which satisfy conditions (2.6) and (2.7). In this situation, compatibility conditions for coring structures on $H$ in the definition of a convolution category, including equations (3.2), are satisfied. For a target of convolution invertible maps take an $R \otimes_{k} L$-ring $A$. In this case the unit maps $\eta_{R}$ and $\eta_{L}$ are obtained as the restrictions of the unit map $R \otimes_{k} L \rightarrow A$ to $R \otimes_{k} 1_{L}$ and to $1_{R} \otimes_{k} L$, respectively. There is no need to distinguish between the products of $A$ as an $R$-ring and as an $L$-ring, so we write simply $\mu_{A}$ for the product in $A$, and it becomes clear from the context, how this should be understood. Since we are dealing with a single product, it makes sense to denote the action of $\mu_{A}$ on elements by juxtaposition. One immediately checks that all the compatibility conditions between the $L-$, and $R$-ring structures on $A$ in the definition of a convolution category are satisfied, in particular (3.1) follow by the associativity of $\mu_{A}$. All this means that, for two bialgebroids $\mathscr{H}_{L}$ and $\mathscr{H}_{R}$ on the same total algebra $H$, such that (2.6) and (2.7) hold, and an $R \otimes_{k} L$-ring $A$, there is a convolution category $\operatorname{Conv}(H, A)$. We can now make explicit the contents of Definition 3.1 in this case. This essentially means describing explicitly all the $L$ and $R$ actions involved.

For left and right bialgebroids $\mathscr{H}_{L}$ and $\mathscr{H}_{R}$ on the same total algebra $H$, such that (2.6) and (2.7) hold, and an $R \otimes_{k} L$-ring $A$, a map $j: H \rightarrow A$ is an $L-R$ bimodule map provided

$$
j\left(s_{L}(l) h s_{R}(r)\right)=\eta_{L}(l) j(h) \eta_{R}(r), \quad \text { for all } l \in L, r \in R, h \in H .
$$

Similarly, $\tilde{j}: H \rightarrow A$ is an $R$ - $L$ bimodule map if

$$
\tilde{j}\left(t_{L}(l) h t_{R}(r)\right)=\eta_{R}(r) \tilde{j}(h) \eta_{L}(l), \quad \text { for all } l \in L, r \in R, h \in H .
$$

A right convolution inverse of $j \in \operatorname{Hom}_{L, R}(H, A)$ is a map $\tilde{j} \in \operatorname{Hom}_{R, L}(H, A)$ such that

$$
\mu_{A} \circ(j \underset{R}{\otimes} \tilde{j}) \circ \gamma_{R}=\eta_{L} \circ \pi_{L} .
$$

A left convolution inverse of $j$ is a map $\hat{j} \in \operatorname{Hom}_{R, L}(H, A)$ such that

$$
\mu_{A} \circ\left(\hat{j} \otimes_{L} j\right) \circ \gamma_{L}=\eta_{R} \circ \pi_{R}
$$

Obviously, by the associativity of the composition in $\operatorname{Conv}(H, A)$, if a map $j: H \rightarrow A$ satisfying (3.3) has both left and right convolution inverses, then they coincide and hence the convolution inverse of an $L-R$ bimodule map $j$ is unique.

Example 3.3. Let $\mathscr{H}_{L}=\left(H, L, s_{L}, t_{L}, \gamma_{L}, \pi_{L}\right)$ be a left bialgebroid and $\mathscr{H}_{R}=\left(H, R, s_{R}, t_{R}, \gamma_{R}\right.$, $\pi_{R}$ ) be a right bialgebroid, on the same total algebra $H$. Assume that the compatibility conditions (2.6) and (2.7) hold. Consider the $R \otimes_{k} L$-ring structure on $H$, defined by the unit map $R \otimes_{k} L \rightarrow H, r \otimes_{k} l \mapsto s_{R}(r) s_{L}(l) \equiv s_{L}(l) s_{R}(r)$. It gives rise to a convolution category Conv $(H, H)$. In light of (3.3), the identity map of $H$ is an element of $\operatorname{Conv}(H, H)(R, L)$. By (3.4), (3.5) and (3.6), the identity map possesses a convolution inverse $S$ if and only if the first equality in (2.8) and (2.9) hold true. Hence it follows by Remark 2.1 that $\left(\mathscr{H}_{L}, \mathscr{H}_{R}, S\right)$ is a Hopf algebroid if and only if $S$ is convolution inverse of the identity map in $\operatorname{Conv}(H, H)$ (in the same way as the antipode of a Hopf algebra $H$ over a ring $k$ is the inverse of the identity map in the convolution algebra $\operatorname{End}_{k}(H)$ ). 
Example 3.4. Example 3.3 can be extended as follows. Take a Hopf algebroid $\mathscr{H}=$ $\left(\mathscr{H}_{L}, \mathscr{H}_{R}, S\right)$ and a left $\mathscr{H}_{L}$-module algebra $B$. (The role of $B$ is played by the base algebra $L$ in Example 3.3.) The smash product algebra $A:=B \rtimes H$ is defined as the $k$-module $B \otimes_{L} H$ with product

$$
(b \rtimes h)\left(b^{\prime} \rtimes h^{\prime}\right):=b\left(h_{(1)} \cdot b^{\prime}\right) \rtimes h_{(2)} h^{\prime},
$$

(cf. [26, Section 2.3]). Here, the left $L$-module structure on $H$ is given by the multiplication by $s_{L}(l)$ on the left. $A$ is an $R$-ring with $\eta_{R}(r)=1_{B} \rtimes s_{R}(r)$ (and hence an $L^{o p}$-ring with unit $\left.l \mapsto 1_{B} \rtimes t_{L}(l)\right)$ and an $L$-ring with unit $\eta_{L}(l)=1_{B} \rtimes s_{L}(l)$. Since the elements $\eta_{R}(r)$ and $\eta_{L}(l)$ commute in $A$, for any $r \in R$ and $l \in L, A$ is an $R \otimes_{k} L$-ring.

The $L-R$ bimodule map $j: H \rightarrow A, h \mapsto 1_{B} \rtimes h$ is convolution invertible with the inverse $j^{\mathrm{c}}: H \rightarrow A, h \mapsto 1_{B} \rtimes S(h)$ (cf. (2.9)).

The notion of a convolution inverse, once established, plays the fundamental role in the definition of a cleft extension of a Hopf algebroid, which we describe presently. Let $\mathscr{H}$ be a Hopf algebroid and $A$ a right $\mathscr{H}$-comodule algebra. Then $A$ is, in particular, an $R$-ring. The unit of this $R$-ring, the algebra homomorphism $R \rightarrow A$, is denoted by $\eta_{R}$. The $k$-subalgebra of $\mathscr{H}_{R}$-coinvariants (whose elements are also $\mathscr{H}_{L}$-coinvariants, cf. Proposition 2.4) in $A$ is denoted by $B$.

Assume that $A$ is also an $L$-ring, with unit $\eta_{L}: L \rightarrow A$, and $B$ is an $L$-subring of $A$. The latter implies that both the $\mathscr{H}_{R}$-coaction $\rho^{A}$, and the $\mathscr{H}_{L}$-coaction $\lambda^{A}$ are left $L$-linear. Since $\rho^{A}$ is $R-R$ bilinear (cf. (2.13)),

$$
\rho^{A}\left(b \eta_{R}(r)\right)=b \underset{R}{\otimes_{R} s_{R}(r)=\rho^{A}\left(\eta_{R}(r) b\right), \quad \text { for all } r \in R, b \in B .}
$$

Thus it follows that $B$ is in the commutant of the image of $\eta_{R}$.

Recall from Section 2.3 that any right $\mathscr{H}$-colinear map $j: H \rightarrow A$ is right $R$-linear in the sense of (3.3) and left $R$-linear in the sense that

$$
j\left(s_{R}(r) h\right)=\eta_{R}(r) j(h),
$$

for all $r \in R$ and $h \in H$ (cf. (2.12)).

Definition 3.5. Let $\mathscr{H}$ be a Hopf algebroid and $A$ a right $\mathscr{H}$-comodule algebra. Denote by $\eta_{R}(r)=r \cdot 1_{A}=1_{A} \cdot r$ the unit map of the corresponding $R$-ring structure of $A$. Let $B$ be the subalgebra of $\mathscr{H}_{R}$-coinvariants in $A$. The extension $B \subseteq A$ is called $\mathscr{H}$-cleft if

(a) $A$ is an $L$-ring (with unit $\eta_{L}: L \rightarrow A$ ) and $B$ is an $L$-subring of $A$;

(b) there exists a convolution invertible left $L$-linear right $\mathscr{H}$-colinear morphism $j: H \rightarrow$ A.

A map $j$, satisfying condition (b), is called a cleaving map.

Condition (b) in Definition 3.5 means, in particular, that a cleaving map is $L-R$ bilinear in the sense of (3.3).

Example 3.6. Consider a smash product algebra $A=B \rtimes H$ of Example 3.4. Similarly to [9. Example 3.7], $A$ is a right $\mathscr{H}$-comodule algebra with $\mathscr{H}_{R^{-}}$-coaction $B \otimes_{L} \gamma_{R}$ and $\mathscr{H}_{L^{-}}$ coaction $B \otimes_{L} \gamma_{L}$. The coinciding subalgebra of $\mathscr{H}_{R}$-coinvariants and $\mathscr{H}_{L}$-coinvariants in $A$ is $B \rtimes 1_{H}$. It is an $L$-subring of $A$. Since the convolution invertible map $j: H \rightarrow A$, $h \mapsto 1_{B} \rtimes h$ in Example 3.4 is right $\mathscr{H}$-colinear, $B \subseteq A$ is an $\mathscr{H}$-cleft extension.

In particular, let $N \subseteq M$ be a depth 2 (or D2, for short) extension of algebras [26, Definition 3.1]. It has been proven in [26, Theorem 4.1] that the algebra $\operatorname{End}_{N, N}(M)$ of $N-N$ 
bilinear endomorphisms of $M$ is a left bialgebroid and $M$ is its left module algebra. By [26, Corollary 4.5], the algebra End ${ }_{-N}(M)$ of right $N$-linear endomorphisms of $M$, with multiplication given by composition, is isomorphic to the smash product algebra $M \rtimes \operatorname{End}_{N, N}(M)$.

If the D2 extension $N \subseteq M$ is also a Frobenius extension then $\operatorname{End}_{N, N}(M)$ is a Hopf algebroid. Hence we can conclude that for any D2 Frobenius extension $N \subseteq M$, the extension $M \subseteq$ End $_{-N}(M)$ (where the inclusion is given by the left multiplication) is a cleft extension of the Hopf algebroid $\operatorname{End}_{N, N}(M)$.

Lemma 3.7. Let $\mathscr{H}$ be a Hopf algebroid and $B \subseteq A$ an $\mathscr{H}$-cleft extension, with a cleaving map $j$. Then

$$
j^{c}\left(t_{R}(r) h\right)=j^{c}(h) \eta_{R}(r), \quad \text { for all } r \in R, h \in H .
$$

Proof. Use the counit property of $\pi_{L}$ (in the first equality), right $L$-linearity of $j^{\text {c }}$, i.e. (3.4) (in the second one), the fact that, since $B$ is an $L$-subring of $A$, the images of $\eta_{L}$ and of $\eta_{R}$ commute in $A$ (in the third one), the assumption that $j^{\mathrm{C}}$ is right convolution inverse of $j$, i.e. (3.5) (in the fourth one), the left $R$ linearity of $j$, i.e. (3.7) (in the fifth one), axiom (2.7) (in the sixth one), the identity $\gamma_{L}\left(s_{R}(r) h\right)=h_{(1)} \otimes_{L} s_{R}(r) h_{(2)}$, for $h \in H$ and $r \in R$, and the assumption that $j^{c}$ is a left convolution inverse of $j$, i.e. (3.6) (in the seventh one), the left $R$-linearity of $j^{\mathrm{c}}$, i.e. (3.4) (in the penultimate one) and the counit property of $\pi_{R}$ (in the last one) to compute

$$
\begin{aligned}
j^{\mathrm{c}}(h) \eta_{R}(r) & =j^{\mathrm{c}}\left(t_{L}\left(\pi_{L}\left(h_{(2)}\right)\right) h_{(1)}\right) \eta_{R}(r)=j^{\mathrm{c}}\left(h_{(1)}\right) \eta_{L}\left(\pi_{L}\left(h_{(2)}\right)\right) \eta_{R}(r) \\
& =j^{\mathrm{c}}\left(h_{(1)}\right) \eta_{R}(r) \eta_{L}\left(\pi_{L}\left(h_{(2)}\right)\right)=j^{\mathrm{c}}\left(h_{(1)}\right) \eta_{R}(r) j\left(h_{(2)}{ }^{(1)}\right) j^{\mathrm{c}}\left(h_{(2)}{ }^{(2)}\right) \\
& =j^{\mathrm{c}}\left(h_{(1)}\right) j\left(s_{R}(r) h_{(2)}{ }^{(1)}\right) j^{\mathrm{c}}\left(h_{(2)}{ }^{(2)}\right)=j^{\mathrm{c}}\left(h^{(1)}(1)\right) j\left(s_{R}(r) h^{(1)}(2)\right) j^{\mathrm{c}}\left(h^{(2)}\right) \\
& =\eta_{R}\left(\pi_{R}\left(s_{R}(r) h^{(1)}\right)\right) j^{\mathrm{c}}\left(h^{(2)}\right)=j^{\mathrm{c}}\left(h^{(2)} t_{R}\left(\pi_{R}\left(s_{R}(r) h^{(1)}\right)\right)\right)=j^{\mathrm{c}}\left(t_{R}(r) h\right),
\end{aligned}
$$

for $h \in H$ and $r \in R$.

In the case of a Hopf algebra cleft extension, the convolution inverse of a cleaving map is a right colinear map, where the right coaction in the Hopf algebra is given by the coproduct followed by the antipode and a flip. In the case of a Hopf algebroid there are two coactions, one for each constituent bialgebroid, related by (2.19). The following lemma shows the behaviour of the convolution inverse of a cleaving map with respect to these right coactions.

Lemma 3.8. Let $\mathscr{H}$ be a Hopf algebroid and $B \subseteq A$ an $\mathscr{H}$-cleft extension with a cleaving map $j$. Then, for all $h \in H$,

$$
\rho^{A}\left(j^{c}(h)\right)=j^{c}\left(h_{(2)}\right) \underset{R}{\otimes} S\left(h_{(1)}\right),
$$

and

$$
\lambda^{A}\left(j^{c}(h)\right)=j^{c}\left(h^{(2)}\right) \underset{L}{\otimes} S\left(h^{(1)}\right) .
$$

Proof. Combining the module map property of the antipode, $S\left(t_{L}(l) h\right)=S(h) s_{L}(l)$, for all $l \in L, h \in H$, with the Hopf algebroid axiom $s_{L}=t_{R} \circ \pi_{R} \circ s_{L}$ and using (3.8), one shows that the expression on the right hand side of (3.9) belongs to the appropriate $R$-module tensor product. Next using (3.4) one finds that it is an element of the Takeuchi product $A \times{ }_{R} H$, defined in (2.11), i.e.

$$
\eta_{R}(r) j^{\mathrm{c}}\left(h_{(2)}\right) \otimes_{R} S\left(h_{(1)}\right)=j^{\mathrm{c}}\left(h_{(2)}\right) \otimes_{R} t_{R}(r) S\left(h_{(1)}\right),
$$


for all $r \in R, h \in H . A \times{ }_{R} H$ is an $R \otimes L$-ring with factorwise multiplication and unit maps

$$
\eta_{R}^{\times}: R \rightarrow A \times{ }_{R} H, \quad r \mapsto 1_{A} \otimes_{R} s_{R}(r) \quad \text { and } \quad \eta_{L}^{\times}: L \rightarrow A \times_{R} H \quad l \mapsto \eta_{L}(l) \otimes_{R}^{1_{H}},
$$

such that $\rho^{A}: A \rightarrow A \times{ }_{R} H$ is a homomorphism of $R \otimes_{k} L$-rings. Furthermore, $\rho^{A} \circ j^{\mathrm{c}}: H \rightarrow$ $A \times{ }_{R} H$ is the convolution inverse of $\rho^{A} \circ j$. We claim that the map

$$
\tilde{\Theta}: H \rightarrow A \times{ }_{R} H, \quad h \mapsto j^{\mathrm{c}}\left(h_{(2)}\right) \otimes_{R} S\left(h_{(1)}\right),
$$

is a right convolution inverse of $\rho^{A} \circ j$.

Take any $h \in H, l \in L$ and $r \in R$. By the Hopf algebroid identity $\gamma_{L}\left(t_{L}(l) h t_{R}(r)\right)=$ $h_{(1)} t_{R}(r) \otimes_{L} t_{L}(l) h_{(2)}$, the module map property of the antipode, $S\left(h t_{R}(r)\right)=s_{R}(r) S(h)$, and the right $L$-linearity of $j^{\mathrm{C}}$ it follows that

$$
\begin{aligned}
\tilde{\Theta}\left(t_{L}(l) h t_{R}(r)\right) & =j^{\mathrm{c}}\left(t_{L}(l) h_{(2)}\right) \bigotimes_{R} S\left(h_{(1)} t_{R}(r)\right) \\
& =j^{\mathrm{c}}\left(h_{(2)}\right) \eta_{L}(l){\underset{R}{R}}_{R}(r) S\left(h_{(1)}\right)=\eta_{R}^{\times}(r) \tilde{\Theta}(h) \eta_{L}(l)^{\times},
\end{aligned}
$$

that is, $\tilde{\Theta}$ satisfies (3.4). Using the right $\mathscr{H}_{R}$-colinearity of $j$ and the coassociativity of $\gamma_{R}$, one computes,

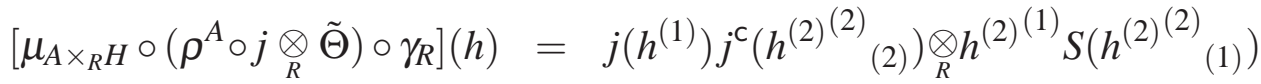

$$
\begin{aligned}
& =j\left(h^{(1)}\right) j^{\mathrm{C}}\left(h^{(2)}{ }_{(2)}\right) \otimes_{R} h^{(2)}{ }_{(1)}^{(1)} S\left(h^{(2)}{ }_{(1)}{ }^{(2)}\right) \\
& =j\left(h^{(1)}\right) j^{\mathrm{c}}\left(h^{(2)}{ }_{(2)}\right){ }_{R}^{\otimes} s_{L}\left(\pi_{L}\left(h^{(2)}(1)\right)\right) \\
& =j\left(h^{(1)}\right) j^{\mathrm{C}}\left(h^{(2)}{ }_{(2)}\right) \eta_{R}\left(\pi_{R}\left(s_{L}\left(\pi_{L}\left(h^{(2)}(1)\right)\right)\right){\underset{R}{H}}_{H}\right. \\
& =j\left(h^{(1)}\right) j^{\complement}\left(s_{L}\left(\pi_{L}\left(h^{(2)}(1)\right)\right) h^{(2)}{ }_{(2)}\right) \otimes_{R} 1_{H}
\end{aligned}
$$

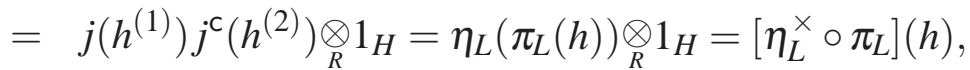

where the second equality follows by the Hopf algebroid axiom (2.7), the third one by the antipode axiom (2.9), the fourth one by the axiom $s_{L}=t_{R} \circ \pi_{R} \circ s_{L}$ in (2.6), the fifth one by (3.8) and the penultimate one by (3.5). Note that (although the counit $\pi_{L}$ is not left $R$-linear), the restriction of $\pi_{L} \otimes_{R} H$ to the Takeuchi product $H \times_{L} H$ is left $R$-linear. Hence all expressions in the above computation are meaningful. This proves that $\tilde{\Theta}$ satisfies $[3.5]$, hence $\tilde{\Theta}$ is a right convolution inverse of $\rho^{A} \circ j$. In view of the uniqueness of a convolution inverse this implies (3.9).

$\mathscr{H}_{L}$-colinearity (i.e. property (3.10) $)$ of $j^{\mathrm{C}}$ is checked by similar steps: The map $R \otimes_{k} L \rightarrow$ $A \times_{L} H, r \otimes_{k} l \mapsto \eta_{L}(l) \otimes_{L} s_{R}(r)$ equips $A \times_{L} H$ with an $R \otimes_{k} L$-ring structure, such that $\lambda^{A}$ : $A \rightarrow A \times{ }_{L} H$ is a morphism of $R \otimes_{k} L$-rings. Moreover, $\lambda^{A} \circ j^{\mathrm{c}}$ is the convolution inverse of $\lambda^{A} \circ j: H \rightarrow A \times_{L} H$, and the map $h \mapsto j^{c}\left(h^{(2)}\right) \otimes_{L} S\left(h^{(1)}\right)$ is checked to be the left convolution inverse of $\lambda^{A} \circ j$. Thus the uniqueness of a convolution inverse implies (3.10).

Remark 3.9. Recall from Section 2.4 that if the antipode of a Hopf algebroid $\mathscr{H}$ is bijective, then there exists an anti-monoidal isomorphism between the categories of right $\mathscr{H}$-comodules and right $\mathscr{H}_{\text {cop }}$-comodules. Hence in this case, in light of the explicit form (2.20) of the relation between the $\mathscr{H}_{L}$ and $\left(\mathscr{H}_{R}\right)_{\text {cop }}$-coactions, an algebra extension $B \subseteq A$ is a right $\mathscr{H}$-extension if and only if $B^{o p} \subseteq A^{o p}$ is a right $\mathscr{H}_{c o p}$-extension. Furthermore, $B \subseteq A$ is an $\mathscr{H}$-cleft extension if and only if $B^{o p} \subseteq A^{o p}$ is an $\mathscr{H}_{c o p}$-cleft extension. Indeed, 
by Lemma 3.8, if $j: H \rightarrow A$ is a cleaving map for the $\mathscr{H}$-cleft extension $B \subseteq A$, then its convolution inverse $j^{\mathrm{C}}$ is a cleaving map for the $\mathscr{H}_{c o p}$-cleft extension $B^{o p} \subseteq A^{o p}$.

Our next aim is to prove that, in parallel to the Hopf algebra case, an $\mathscr{H}$-cleft extension can be equivalently characterised as a Galois extension with the normal basis property. This is the main result of this section. The main difference with the Hopf algebra case is that a cleft $\mathscr{H}$-extension is a Galois extension with respect to the right bialgebroid $\mathscr{H}_{R}$ but it has a normal basis property with respect to the base algebra $L$ of the left bialgebroid $\mathscr{H}_{L}$. In preparation for this we state the following two lemmas.

Lemma 3.10. Let $\mathscr{H}$ be a Hopf algebroid and $B \subseteq A$ an $\mathscr{H}$-cleft extension with a cleaving map $j$. Then, for all $a \in A, a^{[0]} j^{c}\left(a^{[1]}\right) \in B$.

Proof. This is checked by applying $\rho^{A}$ to $a^{[0]} j^{\mathrm{C}}\left(a^{[1]}\right)$, noting that $\rho^{A}$ is an algebra map and $j^{C}$ satisfies equation (3.9), and then repeating the same steps as in the verification that $\tilde{\Theta}$ satisfies equation (3.5) in the proof of Lemma 3.8 .

Lemma 3.11. Let $\mathscr{H}$ be a Hopf algebroid and $B \subseteq A$ an $\mathscr{H}$-cleft extension. Then the inclusion $B \subseteq A$ splits in the category of left $B$-modules. If, in addition, the antipode of $\mathscr{H}$ is bijective, then the inclusion $B \subseteq A$ splits also in the category of right $B$-modules.

Proof. A left $B$-linear splitting of the inclusion $B \rightarrow A$ is given by the map

$$
A \rightarrow B, \quad a \mapsto a^{[0]} j^{\complement}\left(a^{[1]}\right) j\left(1_{H}\right),
$$

where $j$ is a cleaving map. The element $a^{[0]} j^{\mathrm{C}}\left(a^{[1]}\right)$ belongs to $B$ for any $a \in A$ by Lemma 3.10 and $j\left(1_{H}\right)$ is an element of $B$ by the colinearity of $j$ and the unitality of $\rho^{A}$. The left $B$-linearity of the map (3.11) follows by the left $B$-linearity of $\rho^{A}$. Finally, for all $b \in B$,

$$
b^{[0]} j^{\mathrm{c}}\left(b^{[1]}\right) j\left(1_{H}\right)=b j^{\mathrm{c}}\left(1_{H}\right) j\left(1_{H}\right)=b \eta_{R}\left(\pi_{R}\left(1_{H}\right)\right)=b,
$$

where the penultimate equality follows by the fact that $j^{\mathrm{c}}$ is the convolution inverse of $j$ and the unitality of $\gamma_{L}$.

If the antipode is bijective, then, by Remark 3.9, the map

$$
A \rightarrow B, \quad a \mapsto j^{\mathrm{C}}\left(1_{H}\right) j\left(S^{-1}\left(a_{[1]}\right)\right) a_{[0]},
$$

is a right $B$-linear section of the inclusion $B \subseteq A$.

Notice that $B \otimes_{L} H$ is a left $B$-module via the regular $B$-module structure of the first tensor factor, and - since the coproducts $\gamma_{R}$ and $\gamma_{L}$ are left $L$-linear - also a right $\mathscr{H}$-comodule via the regular $\mathscr{H}$ - comodule structure of the second tensor factor.

Theorem 3.12. Let $\mathscr{H}$ be a Hopf algebroid and $B \subseteq A$ a right $\mathscr{H}$-extension. Then the following statements are equivalent:

(1) $B \subseteq A$ is an $\mathscr{H}$-cleft extension.

(a) The extension $B \subseteq A$ is $\mathscr{H}_{R}$-Galois;

(b) $A \simeq B \otimes_{L} H$ as left $B$-modules and right $\mathscr{H}$-comodules.

Proof. $(1) \Rightarrow(2)$ (a) Suppose that $B \subseteq A$ is a cleft $\mathscr{H}$-extension with a cleaving map $j$ and consider the map

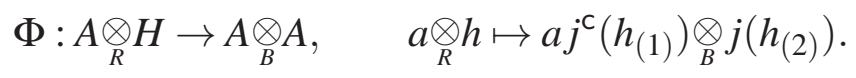


By (3.4) and (3.3), $j^{c}\left(h_{(1)}\right) \otimes_{L} j\left(h_{(2)}\right)$ is a well defined element of $A \otimes_{L} A$. Since $B$ is an $L$-ring, $\Phi$ is a well defined map. We claim that $\Phi$ is the inverse of the $\mathscr{H}_{R}$-canonical map (2.15). Take any $a \otimes_{R} h \in A \otimes_{R} H$ and compute

$$
\begin{aligned}
& \operatorname{can}_{R}\left(\Phi\left(a \otimes_{R} h\right)\right)=a j^{\mathrm{c}}\left(h_{(1)}\right) j\left(h_{(2)}\right)^{[0]}{\underset{R}{\otimes}}_{j} j\left(h_{(2)}\right)^{[1]}=a j^{\mathrm{c}}\left(h_{(1)}\right) j\left(h_{(2)}{ }^{(1)}\right){\underset{R}{(2)}}_{(2)} \\
& =a j^{\mathrm{C}}\left(h^{(1)}{ }_{(1)}\right) j\left(h^{(1)}{ }_{(2)}\right) \underset{R}{\otimes} h^{(2)}=a \eta_{R}\left(\pi_{R}\left(h^{(1)}\right)\right) \underset{R}{\otimes h^{(2)}} \\
& =a \otimes_{R} h^{(2)} t_{R}\left(\pi_{R}\left(h^{(1)}\right)\right)=a \underset{R}{\otimes} h,
\end{aligned}
$$

where the second equality follows by the right $\mathscr{H}_{R}$-colinearity of $j$, the third one by (2.7), and the fourth one by (3.6). On the other hand, for all $a \otimes_{B} a^{\prime} \in A \otimes_{B} A$,

$$
\begin{aligned}
\Phi\left(\operatorname{can}_{R}\left(a \otimes_{B} a^{\prime}\right)\right) & =a a^{\prime[0]} j^{\mathrm{C}}\left(a^{\prime[1]}{ }_{(1)}\right) \bigotimes_{B} j\left(a^{\prime[1]}{ }_{(2)}\right)=a a_{[0]}^{\prime[0]} j^{\mathrm{C}}\left(a_{[0]}^{\prime}{ }^{[1]}\right) \bigotimes_{B} j\left(a_{[1]}^{\prime}\right) \\
& =a \otimes_{B} a_{[0]}^{\prime[0]} j^{\mathrm{C}}\left(a_{[0]}^{\prime}{ }^{[1]}\right) j\left(a_{[1]}^{\prime}\right)=a \otimes_{B} a^{\prime[0]} j^{\mathrm{C}}\left(a^{\prime[1]}{ }_{(1)}\right) j\left(a^{\prime[1]}{ }_{(2)}\right) \\
& =a \bigotimes_{B} a^{\prime[0]} \eta_{R}\left(\pi_{R}\left(a^{\prime[1]}\right)\right)=a \otimes_{B} a^{\prime},
\end{aligned}
$$

where the second and the fourth equalities follow by the right $\mathscr{H}_{L}$-colinearity of $\rho^{A}$, the third one by Lemma 3.10, the fifth one by (3.6) and the last one by the counitality of $\rho^{A}$. Thus $\Phi$ is the inverse of the canonical map, as claimed.

$(1) \Rightarrow(2)(b)$ Given a cleaving map $j$, consider the left $B$-linear map

$$
\kappa: A \rightarrow B \underset{L}{\otimes} H, \quad a \mapsto a_{[0]}^{[0]} j^{\mathrm{c}}\left(a_{[0]}^{[1]}\right) \underset{L}{\otimes} a_{[1]}=a^{[0]} j^{\mathrm{c}}\left(a^{[1]}(1)\right) \underset{L}{\otimes} a^{[1]}{ }_{(2)} .
$$

The equality of two forms of $\kappa$ follows by the right $\mathscr{H}_{L}$-colinearity of $\rho^{A}$. Furthermore, Lemma 3.10 implies that the image of $\kappa$ is in $B \otimes_{L} H$. In the opposite direction, define the left $B$-linear map $v: B \otimes_{L} H \rightarrow A, b \otimes_{L} h \mapsto b j(h)$, which is right $\mathscr{H}$-colinear by the right colinearity of a cleaving map and the left $B$-linearity of the $\mathscr{H}_{R^{-}}$and $\mathscr{H}_{L}$-coactions. The map $v$ is well defined in view of (3.3). For all $b \otimes_{L} h \in B \otimes_{L} H$,

$$
\begin{aligned}
\kappa(v(b \underset{L}{\otimes} h)) & =b j(h)_{[0]}^{[0]} j^{\mathrm{c}}\left(j(h)_{[0]}^{[1]}\right) \underset{L}{\otimes_{L}} j(h)_{[1]}=b j\left(h_{(1)}{ }^{(1)}\right) j^{\mathrm{c}}\left(h_{(1)}{ }^{(2)}\right) \underset{L}{\otimes} h_{(2)} \\
& =b \eta_{L}\left(\pi_{L}\left(h_{(1)}\right)\right){\underset{L}{(2)}}_{h_{(2)}}=b \otimes_{L} s_{L}\left(\pi_{L}\left(h_{(1)}\right)\right) h_{(2)}=b \underset{L}{\otimes} h,
\end{aligned}
$$

where the second equality follows by the $\mathscr{H}$-colinearity of $j$, and the third one by (3.5). On the other hand, (3.6) and the counitality of $\rho^{A}$ imply, for all $a \in A$,

$$
v(\kappa(a))=a^{[0]} j^{\mathrm{c}}\left(a^{[1]}{ }_{(1)}\right) j\left(a^{[1]}{ }_{(2)}\right)=a^{[0]} \eta_{R}\left(\pi_{R}\left(a^{[1]}\right)\right)=a .
$$

This means that $v$ is the left $B$-linear right $\mathscr{H}$-colinear inverse of $\kappa$, hence $\kappa$ is the required isomorphism.

(2) $\Rightarrow$ (1) Suppose that the canonical map (2.15) is bijective and write $\tau=\operatorname{can}_{R}^{-1}\left(1_{A} \otimes_{R}-\right)$ : $H \rightarrow A \otimes_{B} A$ for the translation map. In explicit calculations we use a Sweedler type notation, for all $h \in H, \tau(h)=h^{\{1\}} \otimes_{B} h^{\{2\}}$ (summation understood). Let $\kappa: A \rightarrow B \otimes_{L} H$ be an isomorphism of left $B$-modules and of right $\mathscr{H}$-comodules and define maps $H \rightarrow A$

$$
j:=\kappa^{-1}\left(1_{B} \otimes_{L}^{\otimes}-\right), \quad \tilde{j}:=\left[A \underset{B}{\otimes}\left(B \underset{L}{\otimes} \pi_{L}\right) \circ \kappa\right] \circ \tau .
$$

We claim that $j$ is a cleaving map and $\tilde{j}$ is its convolution inverse. First note that since $\kappa^{-1}$ is left $B$-linear, it is in particular left $L$-linear, hence also $j$ is left $L$-linear. Since $\kappa^{-1}$ is also right $\mathscr{H}$-colinear, so is $j$. Furthermore, the canonical map is left $A$-linear, hence also left $R$-linear. Therefore, its inverse is left $R$-linear, implying that, for all $h \in H$ and 
$r \in R, \tau\left(h t_{R}(r)\right)=\eta_{R}(r) h^{\{1\}} \otimes_{B} h^{\{2\}}$. With this property of the translation map at hand, one immediately finds that, for all $h \in H$ and $r \in R, \tilde{j}\left(h t_{R}(r)\right)=\eta_{R}(r) \tilde{j}(h)$. On the other hand, by (2.13), for all $a, a^{\prime} \in A$ and $r \in R, \operatorname{can}_{R}\left(a \otimes_{B} \eta_{R}(r) a^{\prime}\right)=a a^{\prime[0]} \otimes_{R} s_{R}(r) a^{\prime[1]}$. This implies that $\tau\left(s_{R}(r) h\right)=h^{\{1\}} \otimes_{B} \eta_{R}(r) h^{\{2\}}$. Thus, in view of the Hopf algebroid axiom $t_{L}=s_{R} \circ \pi_{R} \circ t_{L}$, one finds, for all $h \in H$ and $l \in L$,

$$
\tilde{j}\left(t_{L}(l) h\right)=\left[A \underset{B}{\otimes}\left(B \underset{L}{\otimes} \pi_{L}\right) \circ \kappa\right]\left(\tau\left(t_{L}(l) h\right)\right)=\left[A \underset{B}{\otimes}\left(B \underset{L}{\otimes} \pi_{L}\right) \circ \kappa\right]\left(h^{\{1\}} \otimes_{B} \eta_{R}\left(\pi_{R}\left(t_{L}(l)\right)\right) h^{\{2\}}\right) .
$$

Since $\kappa$ is right $\mathscr{H}_{R}$-colinear, it is in particular left $R$-linear, where the left $R$-module structure of $B \otimes_{L} H$ is given by $r \cdot\left(b \otimes_{L} h\right)=b \otimes_{L} s_{R}(r) h$, (cf. (2.12)). By the right $L$-linearity of $\pi_{L}$ and the axiom $t_{L}=s_{R} \circ \pi_{R} \circ t_{L}$, one therefore concludes that $\tilde{j}\left(t_{L}(l) h\right)=\tilde{j}(h) \eta_{L}(l)$, as required. This proves that $\tilde{j}$ satisfies (3.4). It remains to check (3.5) and (3.6):

$$
\begin{aligned}
& \mu_{A} \circ(j \underset{R}{\otimes} \tilde{j}) \circ \gamma_{R}=\mu_{A} \circ\left\{j \underset{R}{\otimes}\left[A \otimes_{B}\left(B \underset{L}{\otimes} \pi_{L}\right) \circ \kappa\right] \circ \tau\right\} \circ \gamma_{R} \\
& =\left[A \underset{B}{\otimes}\left(B \underset{L}{\otimes} \pi_{L}\right) \circ \kappa\right] \circ \operatorname{can}_{R}^{-1} \circ(j \underset{R}{\otimes} H) \circ \gamma_{R} \\
& =\left[A \underset{B}{\otimes}\left(B \underset{L}{\otimes} \pi_{L}\right) \circ \kappa\right] \circ \operatorname{can}_{R}^{-1} \circ \rho^{A} \circ j \\
& =\left[A \underset{B}{\otimes}\left(B \underset{L}{\otimes} \pi_{L}\right) \circ \kappa\right] \circ\left(1_{A} \otimes_{B} j(-)\right)=\eta_{L} \circ \pi_{L},
\end{aligned}
$$

where the second equality follows by the left $A$-linearity of the canonical map $\operatorname{can}_{R}$, hence of $\mathrm{can}_{R}^{-1}$, the third one by the right $\mathscr{H}_{R}$-colinearity of $j$ and the fourth one by the explicit form (2.15) of $\operatorname{can}_{R}$. Furthermore,

$$
\begin{aligned}
& \mu_{A} \circ(\tilde{j} \underset{L}{\otimes} j) \circ \gamma_{L}=\mu_{A} \circ\left\{\left[A \underset{B}{\otimes}\left(B \underset{L}{\otimes} \pi_{L}\right) \circ \kappa\right] \circ \tau_{L}^{\otimes} j\right\} \circ \gamma_{L} \\
& =\mu_{A} \circ\left[A \otimes_{B}\left(B \otimes_{L} \pi_{L}\right) \circ \kappa \otimes_{L} j\right] \circ\left(A \otimes_{B} \lambda^{A}\right) \circ \tau \\
& =\mu_{A} \circ\left(A \underset{B}{\otimes} B \underset{L}{\otimes} \pi_{L} \otimes_{L} j\right) \circ\left(A \underset{B}{\otimes} B \underset{L}{\otimes} \gamma_{L}\right) \circ(A \underset{B}{\otimes} \kappa) \circ \tau \\
& =\mu_{A} \circ[\underset{B}{\otimes} \underset{L}{\otimes}(B \underset{L}{\otimes}) \circ \kappa] \circ \tau=\mu_{A} \circ \tau=\eta_{R} \circ \pi_{R},
\end{aligned}
$$

where the second equality follows by the $\mathscr{H}_{L}$-colinearity of $\tau$, the third one by the $\mathscr{H}_{L^{-}}$ colinearity of $\kappa$, the penultimate one by the left $B$-linearity of $\kappa$ and the last one by $\left(A \underset{R}{\otimes} \pi_{R}\right) \circ$ $\operatorname{can}_{R}=\mu_{A}$ and the definition of the translation map $\tau$.

By Remark 3.9, the following 'left handed version' of Theorem 3.12(1) $\Rightarrow(2)(b)$ can be formulated.

Corollary 3.13. Let $\mathscr{H}$ be a Hopf algebroid with a bijective antipode and $B \subseteq A$ an $\mathscr{H}$ cleft extension with a cleaving map $j$. Then the right $B$-linear left $\mathscr{H}$-colinear map

$$
A \rightarrow H \underset{L}{\otimes} B, \quad a \mapsto S^{-1}\left(a_{[1]}\right)_{(1)} \underset{L}{\otimes} j\left(S^{-1}\left(a_{[1]}\right)_{(2)}\right) a_{[0]} \equiv S^{-1}\left(a^{[1]}\right) \underset{L}{\otimes} j\left(S^{-1}\left(a^{[0]}[1]\right)\right) a^{[0]}[0],
$$

is an isomorphism.

The following is an immediate consequence of Theorem 3.12

Corollary 3.14. Let $\mathscr{H}$ be a Hopf algebroid and $B \subseteq A$ an $\mathscr{H}$-cleft extension. If $H$ is a projective left L-module, then $A$ is a faithfully flat left B-module.

Proof. By Theorem $3.12(1) \Rightarrow(2)(b), A \simeq B \otimes_{L} H$ as left $B$-modules. Since $H$ is projective as a left $L$-module, $A$ is projective as a left $B$-module. Together with Lemma 3.11 this implies the claim. 
If the antipode of a Hopf algebroid $\mathscr{H}$ is bijective then, by [9, Lemma 3.3], an extension $B \subseteq A$ is a right $\mathscr{H}_{R}$-Galois extension if and only if it is a right $\mathscr{H}_{L}$-Galois extension. By [9, Lemma 4.1], this is further equivalent to the left $\mathscr{H}_{R}$-Galois and also to the left $\mathscr{H}_{L}$-Galois property of the extension. Hence repeating the steps in the proof of [11, Proposition 4.1], we conclude that Lemma 3.11, Theorem 3.12 and Remark 3.9 imply the following

Corollary 3.15. Let $\mathscr{H}$ be a Hopf algebroid with a bijective antipode and $B \subseteq A$ an $\mathscr{H}$-cleft extension. Then $A$ is an $R$-relative injective right and left $\mathscr{H}_{R}$-comodule, and an L-relative injective left and right $\mathscr{H}_{L}$-comodule.

\section{CROSSED PROduCTS With Hopf AlGEBRoIdS}

One of the main results in the theory of cleft extensions of Hopf algebras is the equivalent characterisation of such extensions as crossed product algebras with an invertible cocycle (cf. [23, Theorem 11] [7, Theorem 1.18]). The aim of this section is to derive such a characterisation for Hopf algebroid cleft extensions. First we need to develop a suitable theory of crossed products, generalising that of [23] and [6]. We start by extending the notion of a measuring [29, p. 139].

Definition 4.1. Let $\mathscr{L}=(H, L, s, t, \gamma, \pi)$ be a left bialgebroid and $B$ an $L$-ring with unit map $\imath: L \rightarrow B$. $\mathscr{L}$ measures $B$ if there exits a $k$-linear map, called a measuring, $H \otimes_{k} B \rightarrow B$, $h \otimes_{k} b \mapsto h \cdot b$ such that, for all $h \in H, l \in L, b, b^{\prime} \in B$,

(a) $h \cdot 1_{B}=\imath(\pi(h))$;

(b) $(t(l) h) \cdot b=(h \cdot b) \imath(l)$ and $(s(l) h) \cdot b=\imath(l)(h \cdot b)$;

(c) $h \cdot\left(b b^{\prime}\right)=\left(h_{(1)} \cdot b\right)\left(h_{(2)} \cdot b^{\prime}\right)$.

Note that condition (b) means simply that a measuring is an $L-L$ bimodule map, where $H$ is viewed as an $L-L$ bimodule via the left multiplication by $s$ and $t$. A left $\mathscr{L}$-module algebra $B$ is measured by $\mathscr{L}$ with a measuring provided by the left $H$-multiplication in $B$.

Definition 4.2. Let $\mathscr{L}=(H, L, s, t, \gamma, \pi)$ be a left bialgebroid and $\imath: L \rightarrow B$ an $L$-ring, measured by $\mathscr{L}$. A $B$-valued 2-cocycle $\sigma$ on $\mathscr{L}$ is a $k$-linear map $H \otimes_{L^{o p}} H \rightarrow B$ (where the right and left $L^{o p}$-module structures on $H$ are given by right and left multiplication by $t(l)$, respectively) satisfying

(a) $\sigma(s(l) h, k)=\imath(l) \sigma(h, k)$ and $\sigma(t(l) h, k)=\sigma(h, k) \imath(l)$;

(b) $\left(h_{(1)} \cdot l(l)\right) \sigma\left(h_{(2)}, k\right)=\sigma(h, s(l) k)$;

(c) $\sigma(1, h)=\imath(\pi(h))=\sigma(h, 1)$;

(d) $\left[h_{(1)} \cdot \sigma\left(k_{(1)}, m_{(1)}\right)\right] \sigma\left(h_{(2)}, k_{(2)} m_{(2)}\right)=\sigma\left(h_{(1)}, k_{(1)}\right) \sigma\left(h_{(2)} k_{(2)}, m\right)$,

for all $h, k, m \in H, l \in L$.

An $\mathscr{L}$-measured $L$-ring $B$ is called a $\sigma$-twisted left $\mathscr{L}$-module if a 2-cocycle $\sigma$ satisfies

(e) $1_{H} \cdot b=b$,

(f) $\left[h_{(1)} \cdot\left(k_{(1)} \cdot b\right)\right] \sigma\left(h_{(2)}, k_{(2)}\right)=\sigma\left(h_{(1)}, k_{(1)}\right)\left(h_{(2)} k_{(2)} \cdot b\right)$,

for all $h, k \in H, b \in B$.

Conditions (c) in Definition 4.2 determine the normalisation of $\sigma$ and (d) is a cocycle condition. These have the same form as corresponding conditions in the bialgebra case. Conditions (a) determine the module map properties of $\sigma$ while (b) ensures that $\sigma$ is properly $L$-balanced; both are needed for (d) (and (f)) to make sense. Condition (e) sates that a measuring is a weak action (cf. [6, Definition 1.1]). 
Similarly to the bialgebra case, the map $\sigma\left(h, h^{\prime}\right):=\imath\left(\pi\left(h h^{\prime}\right)\right)$ is a (trivial) cocycle for an $\mathscr{L}$-measured $L$-ring $B$ with unit $l$, provided that the measuring restricts to the action on $L$, $h \cdot \imath(l)=\imath(\pi(h s(l)))$, for $h \in H$ and $l \in L$. A twisted left $\mathscr{L}$-module corresponding to this trivial cocycle $\sigma$ is simply a left $\mathscr{L}$-module algebra.

Proposition 4.3. Let $\mathscr{L}=(H, L, s, t, \gamma, \pi)$ be a left bialgebroid and $\imath: L \rightarrow B$ an $L$-ring, measured by $\mathscr{L}$. Let $\sigma: H \otimes_{L^{o p}} H \rightarrow B$ be a map, satisfying properties $(a)$ and $(b)$ in Definition 4.2 Consider the k-module $B \otimes_{L} H$, where the left L-module structure on $H$ is given by multiplication by $s(l)$ on the left. $B \otimes_{L} H$ is an associative algebra with unit $1_{B} \otimes_{L} 1_{H}$ and product

$$
(B \underset{L}{\otimes} H) \underset{k}{\otimes}(B \underset{L}{\otimes} H) \rightarrow(B \underset{L}{\otimes} H), \quad(b \underset{L}{\otimes} h) \underset{k}{\otimes}\left(b^{\prime} \underset{L}{\otimes} h^{\prime}\right) \mapsto b\left(h_{(1)} \cdot b^{\prime}\right) \sigma\left(h_{(2)}, h_{(1)}^{\prime}\right) \underset{L}{\otimes} h_{(3)} h_{(2)}^{\prime},
$$

if and only if $\sigma$ is a cocycle and $B$ is a $\sigma$-twisted $\mathscr{L}$-module. The resulting associative algebra is called a crossed product of $B$ with $\mathscr{L}$ and is denoted by $B^{\#} \sigma \mathscr{L}$.

Note that the smash product algebra in Example 3.4 is a crossed product with a trivial cocycle.

Proof. The element $1_{B}^{\# 1} 1_{H}$ is a left unit if and only if

$$
b \# h=\left(1_{H} \cdot b\right) \sigma\left(1_{H}, h_{(1)}\right) \# h_{(2)}, \quad \text { for all } b \# h \in \underset{L}{\otimes} H .
$$

If $\sigma\left(1_{H}, h\right)=\imath(\pi(h))$ and $1_{H} \cdot b=b$, then (4.2) obviously holds. On the other hand, applying $B \otimes_{L} \pi$ to (4.2) we arrive at the identity

$$
b \imath(\pi(h))=\left(1_{H} \cdot b\right) \sigma\left(1_{H}, h\right), \quad \text { for all } b \in B, h \in H .
$$

Setting $b=1_{B}$ in (4.3) we obtain $\sigma\left(1_{H}, h\right)=\imath(\pi(h))$, and setting $h=1_{H}$ we get $1_{H}$. $b=b$. Analogously, the condition that $1_{B}^{\# 1_{H}}$ is a right unit is equivalent to the condition $\sigma\left(h, 1_{H}\right)=\imath(\pi(h))$, for all $h \in H$.

The associative law for product (4.1) reads, for all $h, k, m \in H, a, b, c \in B$,

$$
\begin{aligned}
& a\left(h_{(1)} \cdot b\right) \sigma\left(h_{(2)}, k_{(1)}\right)\left(h_{(3)} k_{(2)} \cdot c\right) \sigma\left(h_{(4)} k_{(3)}, m_{(1)}\right) \# h_{(5)} k_{(4)} m_{(2)}= \\
& a\left(h_{(1)} \cdot b\right)\left[h_{(2)} \cdot\left(k_{(1)} \cdot c\right)\right]\left[h_{(3)} \cdot \sigma\left(k_{(2)}, m_{(1)}\right)\right] \sigma\left(h_{(4)}, k_{(3)} m_{(2)}\right) \# h_{(5)} k_{(4)} m_{(3)} .
\end{aligned}
$$

If $\sigma$ is a cocycle and $B$ is a $\sigma$-twisted module, then (4.4) obviously holds. Note that, for all $h, k \in H$,

$$
\sigma\left(h_{(1)}, k_{(1)}\right) \imath\left(\pi\left(h_{(2)} k_{(2)}\right)\right)=\sigma(h, k) .
$$

Applying $B \otimes_{L} \pi$ to (4.4), using (4.5) and setting $a=1_{B}=b$, we arrive at

$$
\sigma\left(h_{(1)}, k_{(1)}\right)\left(h_{(2)} k_{(2)} \cdot c\right) \sigma\left(h_{(3)} k_{(3)}, m\right)=\left[h_{(1)} \cdot\left(k_{(1)} \cdot c\right) \sigma\left(k_{(2)}, m_{(1)}\right)\right] \sigma\left(h_{(2)}, k_{(3)} m_{(2)}\right) .
$$

Setting $c=1_{B}$ in (4.6) we derive the cocycle condition Definition 4.2 (d), while setting $m=1_{H}$ in (4.6) we obtain Definition 4.2(f).

Theorem 4.4. Let $\mathscr{L}=(H, L, s, t, \gamma, \pi)$ be a left bialgebroid and $\imath: L \rightarrow B$ an $L$-ring. View $A=B \otimes_{L} H$ as a left $B$-module and a right $\mathscr{L}$-comodule in canonical ways (i.e. the left $B$-multiplication is given by product in $B$ and the right $\mathscr{L}$-coaction is $B \otimes_{L} \gamma$, with the Lactions on $H$ given by the left multiplication by $s$ and $t)$. Then $A$ is a right $\mathscr{L}$-comodule algebra with unit $1_{B} \otimes_{L} 1_{H}$ and a left $B$-linear product if and only if $A$ is a crossed product algebra as in Proposition 4.3 . 
Proof. The definition of the product in $B^{\#}{ }_{\sigma} \mathscr{L}$ immediately implies that $B^{\#}{ }_{\sigma} \mathscr{L}$ is a right $\mathscr{L}$-comodule algebra with a left $B$-linear multiplication. Conversely, suppose that $A$ has the required $\mathscr{L}$-comodule algebra structure. Then, in particular, $A$ is an $L^{o p}$-ring via $L^{o p} \rightarrow A$, $l \mapsto 1_{B} \otimes_{L} t(l)$. We use the hom-tensor relation

$$
\operatorname{Hom}_{B-}^{-\mathscr{L}}\left((B \underset{L}{\otimes} H) \underset{L^{o p}}{\otimes}(B \underset{L}{\otimes} H), B \underset{L}{\otimes} H\right) \simeq \operatorname{Hom}_{L, L}\left(H_{L^{o p}}^{\otimes}(B \underset{L}{\otimes} H), B\right)
$$

and the $\mathscr{L}$-colinearity of the product in $A$, to view the multiplication in $A$ as an $L-L$ bilinear map $H \otimes_{L^{o p}}\left(B \otimes_{L} H\right) \rightarrow B$. For any $b \in B$ and $h \in H$, define

$$
h \cdot b:=(B \underset{L}{\otimes} \pi)\left(\left(1_{B} \underset{L}{\otimes} h\right)\left(b \underset{L}{\otimes} 1_{H}\right)\right) .
$$

By (4.7), the above definition implies that, conversely,

$$
\left(1_{B} \underset{L}{\otimes} h\right)\left(b \underset{L}{\otimes} 1_{H}\right)=h_{(1)} \cdot b \underset{L}{\otimes} h_{(2)} .
$$

Now, the assumption that $1_{B} \otimes_{L} 1_{H}$ is the unit in $A$ implies condition (a) in Definition 4.1 The conditions (b) follow by the right $L$-linearity and the left $B$-linearity of the product respectively (remember that every right $\mathscr{L}$-comodule map is necessarily right $L$-linear). The condition (c) follows by the associativity of the product. Thus $B$ is measured by $\mathscr{L}$ with measuring (4.8).

Next, for all $h, h^{\prime} \in H$, define

$$
\sigma\left(h, h^{\prime}\right):=(B \underset{L}{\otimes} \pi)\left(\left(1_{B} \underset{L}{\otimes} h\right)\left(1_{B} \underset{L}{\otimes} h^{\prime}\right)\right) .
$$

Then, by (4.7),

$$
\left(1_{B} \underset{L}{\otimes} h\right)\left(1_{B} \underset{L}{\otimes} h^{\prime}\right)=\sigma\left(h_{(1)}, h_{(1)}^{\prime}\right) \underset{L}{\otimes} h_{(2)} h_{(2)}^{\prime} .
$$

Since $A$ is an $L^{o p}$-ring, (4.10) defines a $k$-linear map $\sigma: H \otimes_{L^{o p}} H \rightarrow B$. The conditions (a) in Definition 4.2 follow by the left $B$-linearity and the right $L$-linearity of the product respectively. To check condition (b), take any $h, k \in H$ and $l \in L$, and compute

$$
\begin{aligned}
\left(h_{(1)} \cdot l(l)\right) \sigma\left(h_{(2)}, k\right) & =(B \underset{L}{\otimes} \pi)\left[\left(1_{B} \otimes_{L} h\right)\left(l(l) \bigotimes_{L}^{\otimes} 1_{H}\right)\left(1_{B} \otimes_{L}^{\otimes} k\right)\right] \\
& =\left(B \otimes_{L}^{\otimes} \pi\right)\left[\left(1_{B} \otimes_{L} h\right)\left(1_{B} \bigotimes_{L} s(l) k\right)\right]=\sigma(h, s(l) k),
\end{aligned}
$$

where the first and last equalities follow by the definitions of the measuring and $\sigma$ and equations (4.9), (4.11), and the left $B$-linearity of the product. Finally, for all $b, b^{\prime} \in B$, $h, h^{\prime} \in H$,

$$
\begin{aligned}
(b \underset{L}{\otimes} h)\left(b^{\prime} \otimes_{L} h^{\prime}\right) & =b\left[\left(1_{B} \underset{L}{\otimes} h\right)\left(b^{\prime} \otimes_{L} 1_{H}\right)\left(1_{B} \underset{L}{\otimes} h^{\prime}\right)\right] \\
& =b\left(h_{(1)} \cdot b^{\prime}\right)\left[\left(1_{B}{ }_{L} h_{(2)}\right)\left(1_{B} \underset{L}{\otimes} h^{\prime}\right)\right]=b\left(h_{(1)} \cdot b^{\prime}\right) \sigma\left(h_{(2)}, h_{(1)}^{\prime}\right) \underset{L}{\otimes} h_{(3)} h_{(2)}^{\prime},
\end{aligned}
$$

where we have used the left $B$-linearity of the product and (4.9) and (4.11). Proposition 4.3 yields the assertion.

Corollary 4.5. Given a crossed product $B^{\#} \sigma \mathscr{L}$ and a convolution invertible map $\chi \in$ $\operatorname{Hom}_{L, L}(H, B)$ such that $\chi\left(1_{H}\right)=1_{B}$, define, for all $h, k \in H$ and $b \in B$,

$$
\begin{gathered}
h \cdot \chi b:=\chi\left(h_{(1)}\right)\left(h_{(2)} \cdot b\right) \chi^{c}\left(h_{(3)}\right), \\
\sigma^{\chi}(h, k):=\chi\left(h_{(1)}\right)\left(h_{(2)} \cdot \chi\left(k_{(1)}\right)\right) \sigma\left(h_{(3)}, k_{(2)}\right) \chi^{c}\left(h_{(4)} k_{(3)}\right) .
\end{gathered}
$$

Then $B$ is a $\sigma^{\chi}$-twisted $\mathscr{L}$-module with measuring (4.12). The corresponding crossed product $B^{\#}{ }_{\sigma} \chi \mathscr{L}$ is called a gauge transform of $B^{\#} \sigma \mathscr{L}$. 
Proof. Any convolution invertible map $\chi \in \operatorname{Hom}_{L, L}(H, B)$ defines a left $B$-module right $\mathscr{L}$-comodule automorphism $\Phi$ of $B \otimes_{L} H$, by

$$
\Phi\left(b \otimes_{L} h\right)=b \chi\left(h_{(1)}\right) \otimes_{L} h_{(2)}, \quad \Phi^{-1}(b \underset{L}{\otimes} h)=b \chi^{\mathrm{c}}\left(h_{(1)}\right) \otimes_{L} h_{(2)} .
$$

If $\chi\left(1_{H}\right)=1_{B}$, then $\Phi\left(1_{H} \otimes_{L} 1_{B}\right)=1_{H} \otimes_{L} 1_{B}$. We can use this isomorphism to induce a new right $\mathscr{L}$-comodule algebra structure on $B \otimes_{L} H$ (with unit $1_{H} \otimes_{L} 1_{B}$ ) from that of $B{ }^{\#} \sigma \mathscr{L}$. In view of Theorem 4.4 this necessarily is a crossed product with the measuring and cocycle given by equations (4.8) and (4.10), i.e., for all $b \in B$ and $h, k \in H$,

$$
\begin{gathered}
h \cdot \chi b=(B \underset{L}{\otimes} \pi)\left(\Phi^{-1}\left(\Phi\left(1_{B} \underset{L}{\otimes} h\right) \Phi\left(b \underset{L}{\otimes} 1_{H}\right)\right)\right), \\
\sigma^{\chi}(h, k)=(B \underset{L}{\otimes} \pi)\left(\Phi^{-1}\left(\Phi\left(1_{B} \otimes_{L} h\right) \Phi\left(1_{B} \otimes_{L} k\right)\right)\right),
\end{gathered}
$$

where the product is computed in $B^{\#} \sigma \mathscr{L}$. One easily checks that these have the form stated in equations (4.12) and (4.13).

Definition 4.6. Let $\mathscr{L}=(H, L, s, t, \gamma, \pi)$ be a left bialgebroid and $B$ an $L$-ring. Crossed products $B^{\#_{\sigma}} \mathscr{L}$ and $B^{\#} \bar{\sigma} \mathscr{L}$ are said to be equivalent if there exists a left $B$-module isomorphism of right $\mathscr{L}$-comodule algebras $B^{\#} \bar{\sigma} \mathscr{L} \rightarrow B^{\#} \sigma \mathscr{L}$.

Theorem 4.7. Let $\mathscr{L}=(H, L, s, t, \gamma, \pi)$ be a left bialgebroid and $B$ an L-ring. Crossed products $B^{\#}{ }_{\sigma} \mathscr{L}$ and $B^{\#} \bar{\sigma}^{L} \mathscr{L}$ are equivalent if and only if $B{ }^{\#} \mathscr{L}$ is a gauge transform of $B^{B} \sigma \mathscr{L}$.

Proof. In view of the hom-tensor relation $\operatorname{Hom}_{B-}^{-\mathscr{L}}\left(B \otimes_{L} H, B \otimes_{L} H\right) \simeq \operatorname{Hom}_{L, L}(H, B)$, there is a bijective correspondence between left $B$-module right $\mathscr{L}$-comodule isomorphisms $\Phi: B{ }^{\#}{ }_{\bar{\sigma}} \mathscr{L} \rightarrow B^{\#_{\sigma}} \mathscr{L}$ and convolution invertible maps $\chi \in \operatorname{Hom}_{L, L}(H, B)$. This correspondence is given by equation (4.14) in one direction and by $\chi(h)=\left(B \otimes_{L} \pi\right)\left(\Phi\left(1_{B} \otimes_{L} h\right)\right)$ in the other. If $\Phi$ is also an algebra map, then $\chi\left(1_{H}\right)=1_{B}$ and, following the same line of argument as in the proof of Corollary 4.5 , one finds that the measuring corresponding to $\bar{\sigma}$ is given by $h \cdot \chi b$ and that $\bar{\sigma}=\sigma^{\chi}$. Conversely, given $\chi$ and corresponding (by equations (4.14) isomorphism $\Phi: B^{\#}{ }_{\sigma \chi} \mathscr{L} \rightarrow B^{\#}{ }_{\sigma} \mathscr{L}$, one can compute, for all $b, b^{\prime} \in B, h, h^{\prime} \in H$,

$$
\begin{aligned}
\Phi\left(\left(b_{\sigma} \#_{\alpha} h\right)\right. & \left.\left(b^{\prime} \#_{\sigma} \chi h^{\prime}\right)\right)=b\left(h_{(1)} \cdot \chi b^{\prime}\right) \sigma^{\chi}\left(h_{(2)}, h_{(1)}^{\prime}\right) \chi\left(h_{(3)} h_{(2)}^{\prime}\right) \#_{\sigma} h_{(4)} h_{(3)}^{\prime} \\
& =b \chi\left(h_{(1)}\right)\left(h_{(2)} \cdot b^{\prime}\right)\left(h_{(3)} \cdot \chi\left(h_{(1)}^{\prime}\right)\right) \sigma\left(h_{(4)}, h_{(2)}^{\prime}\right) \#_{\sigma} h_{(5)} h_{(3)}^{\prime} \\
& =b \chi\left(h_{(1)}\right)\left(h_{(2)} \cdot\left(b^{\prime} \chi\left(h^{\prime}{ }_{(1)}\right)\right) \sigma\left(h_{(3)}, h_{(2)}^{\prime}\right) \#_{\sigma} h_{(4)} h_{(3)}^{\prime}=\Phi\left(b \#_{\sigma} \chi h\right) \Phi\left(b^{\prime} \#_{\sigma} \chi h^{\prime}\right),\right.
\end{aligned}
$$

where the second equality follows by the fact that $\chi^{\mathrm{c}}$ is the convolution inverse of $\chi$ and the counit axiom, and the third equality follows by property (c) in Definition 4.1. This proves that $\Phi$ is an algebra map, hence the crossed product algebras $B^{\#_{\sigma}} \chi \mathscr{L}$ and $B^{\#_{\sigma}} \mathscr{L}$ are mutually equivalent.

Next we establish what is meant by an invertible cocycle in this generalised context.

Definition 4.8. Let $\mathscr{L}=(H, L, s, t, \gamma, \pi)$ be a left bialgebroid and $\imath: L \rightarrow B$ an $L$-ring, measured by $\mathscr{L}$. A $B$-valued 2-cocycle $\sigma$ on $\mathscr{L}$ is invertible if there exists a $k$-linear map $\tilde{\sigma}: H \otimes_{L} H \rightarrow B$ (where the right and left $L$-module structures on $H$ are given by right and left multiplication by $s(l)$, respectively) satisfying

(a) $\tilde{\sigma}(s(l) h, k)=\imath(l) \tilde{\sigma}(h, k)$ and $\tilde{\sigma}(t(l) h, k)=\tilde{\sigma}(h, k) \imath(l)$;

(b) $\tilde{\sigma}\left(h_{(1)}, k\right)\left(h_{(2)} \cdot \imath(l)\right)=\tilde{\sigma}(h, t(l) k)$;

(c) $\sigma\left(h_{(1)}, k_{(1)}\right) \tilde{\sigma}\left(h_{(2)}, k_{(2)}\right)=h \cdot\left(k \cdot 1_{B}\right)$ and $\tilde{\sigma}\left(h_{(1)}, k_{(1)}\right) \sigma\left(h_{(2)}, k_{(2)}\right)=h k \cdot 1_{B}$, 
for all $h, k \in H$ and $l \in L$. A map $\tilde{\sigma}$ is called an inverse of $\sigma$.

Again, conditions (a) and (b) are needed so that the inverse property (c) can be stated. In the case $\mathscr{L}$ is a bialgebra over a ring $L=k$, conditions (a) and (b) are satisfied automatically. The following two lemmas explore the nature of cocycles and their inverses.

Lemma 4.9. Let $\mathscr{L}=(H, L, s, t, \gamma, \pi)$ be a left bialgebroid and $B$ an L-ring with unit $\imath$ : $L \rightarrow B$. Assume that $\mathscr{L}$ measures $B$ and $\sigma$ is an invertible $B$-valued 2-cocycle on $\mathscr{L}$. Then an inverse $\tilde{\sigma}$ of $\sigma$ is unique and normalised, i.e., for all $h \in H$,

$$
\tilde{\sigma}\left(1_{H}, h\right)=\imath(\pi(h))=\tilde{\sigma}\left(h, 1_{H}\right) .
$$

Proof. Note that, if $\tilde{\sigma}$ is an inverse of $\sigma$, then, for all $h, k \in H$,

$$
\imath\left(\pi\left(h_{(1)} k_{(1)}\right)\right) \tilde{\sigma}\left(h_{(2)}, k_{(2)}\right)=\tilde{\sigma}(h, k) .
$$

Using this identity and Definition 4.8 (c), one finds that

$$
\tilde{\sigma}(h, k)=\tilde{\sigma}\left(h_{(1)}, k_{(1)}\right)\left[h_{(2)} \cdot\left(k_{(2)} \cdot 1_{B}\right)\right] .
$$

Now suppose that $\hat{\sigma}$ is another inverse of $\sigma$. Then replacing the expression in square brackets in (4.16) by the first of equations in Definition 4.8 (c) for $\hat{\sigma}$, using the second of equations Definition 4.8 (c) for $\tilde{\sigma}$, and finally using (4.15) for $\hat{\sigma}$, one finds that $\tilde{\sigma}=\hat{\sigma}$. Hence the inverse of a cocycle is unique.

Use (4.15), Definition 4.2 and Definition4.8 (c) to compute, for all $h \in H$,

$$
\begin{aligned}
\tilde{\sigma}\left(1_{H}, h\right) & =\imath\left(\pi\left(h_{(1)}\right)\right) \tilde{\sigma}\left(1_{H}, h_{(2)}\right)=\sigma\left(1_{H}, h_{(1)}\right) \tilde{\sigma}\left(1_{H}, h_{(2)}\right) \\
& =\left[1_{H} \cdot\left(h \cdot 1_{B}\right)\right] \sigma\left(1_{H}, 1_{H}\right)=\sigma\left(1_{H}, s(\pi(h))\right)=\imath(\pi(h)) .
\end{aligned}
$$

The proof of the other identity is similar.

Lemma 4.10. Let $\mathscr{L}=(H, L, s, t, \gamma, \pi)$ be a left bialgebroid, $B$ an L-ring, measured by $\mathscr{L}$, and $\sigma$ an invertible $B$-valued 2-cocycle on $\mathscr{L}$ with the inverse $\tilde{\sigma}$. For all $h, k, m \in H$,

(a) $h \cdot \sigma(k, m)=\sigma\left(h_{(1)}, k_{(1)}\right) \sigma\left(h_{(2)} k_{(2)}, m_{(1)}\right) \tilde{\sigma}\left(h_{(3)}, k_{(3)} m_{(2)}\right)$,

(b) $h \cdot \tilde{\sigma}(k, m)=\sigma\left(h_{(1)}, k_{(1)} m_{(1)}\right) \tilde{\sigma}\left(h_{(2)} k_{(2)}, m_{(2)}\right) \tilde{\sigma}\left(h_{(3)}, k_{(3)}\right)$.

Proof. (a) Denote the unit map of the $L$-ring $B$ by $\imath: L \rightarrow B$. In view of (4.5) and with the help of properties (c) and (a) in Definition 4.1 and (c) in Definition 4.8, we can compute, for all $h, k, m \in H$,

$$
\begin{aligned}
h \cdot \sigma(k, m) & =h \cdot\left[\sigma\left(k_{(1)}, m_{(1)}\right) \imath\left(\pi\left(k_{(2)} m_{(2)}\right)\right)\right]=\left[h_{(1)} \cdot \sigma\left(k_{(1)}, m_{(1)}\right)\right]\left[h_{(2)} \cdot\left(k_{(2)} m_{(2)} \cdot 1_{B}\right)\right] \\
& =\left[h_{(1)} \cdot \sigma\left(k_{(1)}, m_{(1)}\right)\right] \sigma\left(h_{(2)}, k_{(2)} m_{(2)}\right) \tilde{\sigma}\left(h_{(3)}, k_{(3)} m_{(3)}\right) \\
& =\sigma\left(h_{(1)}, k_{(1)}\right) \sigma\left(h_{(2)} k_{(2)}, m_{(1)}\right) \tilde{\sigma}\left(h_{(3)}, k_{(3)} m_{(2)}\right),
\end{aligned}
$$

where the last equality follows by property (d) in Definition 4.2 .

(b) Use part (a), (4.5) and Definition 4.8 (c) to find that, for all $h, k, m \in H$,

$$
\begin{aligned}
{\left[h_{(1)} \cdot \sigma\left(k_{(1)}, m_{(1)}\right)\right] } & \sigma\left(h_{(2)}, k_{(2)} m_{(2)}\right) \tilde{\sigma}\left(h_{(3)} k_{(3)}, m_{(3)}\right) \tilde{\sigma}\left(h_{(4)}, k_{(4)}\right) \\
& =\sigma\left(h_{(1)}, k_{(1)}\right)\left[h_{(2)} k_{(2)} \cdot\left(m \cdot 1_{B}\right)\right] \tilde{\sigma}\left(h_{(3)}, k_{(3)}\right) .
\end{aligned}
$$

By Definitions 4.1 and 4.2 $\left.\sigma(h, s(\pi(m)))=h \cdot\left(m \cdot 1_{B}\right)\right)$, for all $h, m \in H$. Hence conditions (d) in Definition 4.2 and (c) in Definition 4.8 allow us to develop the right hand side of the above equality further to arrive at the equation

$$
\left[h_{(1)} \cdot \sigma\left(k_{(1)}, m_{(1)}\right)\right] \sigma\left(h_{(2)}, k_{(2)} m_{(2)}\right) \tilde{\sigma}\left(h_{(3)} k_{(3)}, m_{(3)}\right) \tilde{\sigma}\left(h_{(4)}, k_{(4)}\right)=h \cdot\left[k \cdot\left(m \cdot 1_{B}\right)\right] .
$$


Therefore

$$
\begin{aligned}
& h \cdot \tilde{\sigma}(k, m)=h \cdot\left\{\tilde{\sigma}\left(k_{(1)}, m_{(1)}\right)\left[k_{(2)} \cdot\left(m_{(2)} \cdot 1_{B}\right)\right]\right\} \\
& =\left[h_{(1)} \cdot \tilde{\sigma}\left(k_{(1)}, m_{(1)}\right)\right]\left\{h_{(2)} \cdot\left[k_{(2)} \cdot\left(m_{(2)} \cdot 1_{B}\right)\right]\right\} \\
& =\left[h_{(1)} \cdot \tilde{\sigma}\left(k_{(1)}, m_{(1)}\right)\right]\left[h_{(2)} \cdot \sigma\left(k_{(2)}, m_{(2)}\right)\right] \sigma\left(h_{(3)}, k_{(3)} m_{(3)}\right) \tilde{\sigma}\left(h_{(4)} k_{(4)}, m_{(4)}\right) \tilde{\sigma}\left(h_{(5)}, k_{(5)}\right) \\
& =\left[h_{(1)} \cdot\left(k_{(1)} m_{(1)} \cdot 1_{B}\right)\right] \sigma\left(h_{(2)}, k_{(2)} m_{(2)}\right) \tilde{\sigma}\left(h_{(3)} k_{(3)}, m_{(3)}\right) \tilde{\sigma}\left(h_{(4)}, k_{(4)}\right) \\
& =\sigma\left(h_{(1)}, k_{(1)} m_{(1)}\right) \tilde{\sigma}\left(h_{(2)} k_{(2)}, m_{(2)}\right) \tilde{\sigma}\left(h_{(3)}, k_{(3)}\right),
\end{aligned}
$$

where the first equality follows by (4.16), the second one by property (c) in Definition 4.1 and the third one by (4.17). The penultimate equality follows by property (c) in Definition 4.1 and (c) in Definition 4.8. The last equality follows by conditions (a) in Definition 4.1 and (b) in Definition 4.2 .

Take a Hopf algebroid $\mathscr{H}$, an $\mathscr{H}_{L}$-measured $L$-ring $B$ and a cocycle $\sigma$. Then the crossed product $B^{\#} \sigma \mathscr{H}_{L}$ is an $R$-ring with unit map $\eta_{R}: r \mapsto 1_{B}{ }^{\#} S_{R}(r)$ and an $L$-ring with unit map $\eta_{L}: l \mapsto 1_{B}^{\# S_{L}}(l)=l(l) \# 1_{H}$, where $l: L \rightarrow B$ denotes the unit map of the $L$-ring $B$. It is also a right $\mathscr{H}$-comodule with $\mathscr{H}_{R^{-}}$-coaction $B \otimes_{L} \gamma_{R}$ and right $\mathscr{H}_{L}$-coaction $B \otimes_{L} \gamma_{L}$. The $\mathscr{H}_{R^{-}}$ coinvariants are the elements of the form $b \# 1_{H}$ for $b \in B$, hence they form an $L$-subring, isomorphic to $B$. Therefore, $B \subseteq B^{\#} \sigma \mathscr{H}_{L}$ is a right $\mathscr{H}$-extension, and it is natural to ask whether this extension is cleft.

Theorem 4.11. Let $\mathscr{H}$ be a Hopf algebroid and $B^{\#} \sigma \mathscr{H}_{L}$ a crossed product of an $\mathscr{H}_{L^{-}}$ measured $L$-ring $B$. If the cocycle $\sigma$ is invertible, then the extension $B \subseteq B \#_{\sigma} \mathscr{H}_{L}$ is $\mathscr{H}$-cleft.

Proof. We claim that the right $\mathscr{H}$-comodule map $j: H \rightarrow B{ }^{\#} \sigma \mathscr{H}_{L}, h \mapsto 1_{B} \# h$ is a cleaving map with the convolution inverse

$$
j^{\complement}(h)=\tilde{\sigma}\left(S\left(h_{(1)}\right)_{(1)}, h_{(2)}\right) \# S\left(h_{(1)}\right)_{(2)}=\tilde{\sigma}\left(S\left(h_{(1)}^{(2)}\right), h_{(2)}^{(2)}\right) \# S\left(h^{(1)}\right) .
$$

The two forms of $j^{\mathrm{c}}$ are equivalent by the anti-comultiplicativity of $S$ and the left $\mathscr{H}_{R^{-}}$ colinearity of $\gamma_{L}$. Using the definitions of a cocycle and its inverse, and in particular, the module and normalisation properties of $\sigma$ and $\tilde{\sigma}$, one verifies that $j$ and $j^{\mathrm{c}}$ have the required $L-, R$-module map properties (3.3) and (3.4). Next, take any $h \in H$ and compute

$$
\begin{aligned}
& j^{\mathrm{C}}\left(h_{(1)}\right) j\left(h_{(2)}\right)=\tilde{\sigma}\left(S\left(h_{(1)}\right)_{(1)}, h_{(2)}\right) \sigma\left(S\left(h_{(1)}\right)_{(2)}, h_{(3)}\right) \# S\left(h_{(1)}\right)_{(3)} h_{(4)}
\end{aligned}
$$

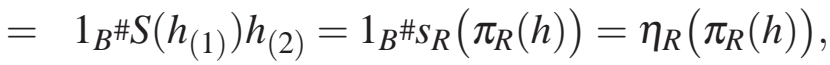

where the second equality follows by condition (c) in Definition 4.8, condition (a) in Definition 4.1 and the counit property of $\pi_{L}$. The third equality follows by the antipode axiom 
(2.9). The proof of the identity (3.5) is slightly more involved:

$$
\begin{aligned}
& j\left(h^{(1)}\right) j^{\mathrm{C}}\left(h^{(2)}\right) \\
& =\left[h^{(1)}{ }_{(1)} \cdot \tilde{\sigma}\left(S\left(h^{(2)}{ }_{(1)}\right)_{(1)}, h_{(2)}^{(2)}\right)\right] \sigma\left(h_{(2)}^{(1)}, S\left(h_{(1)}^{(2)}\right)_{(2)}\right) \# h_{(3)}^{(1)} S\left(h^{(2)}{ }_{(1)}\right)_{(3)} \\
& =\sigma\left(h_{(1)}^{(1)}, S\left(h_{(1)}^{(2)}\right)_{(1)} h_{(2)}^{(2)}\right) \tilde{\sigma}\left(h^{(1)}{ }_{(2)} S\left(h_{(1)}^{(2)}\right)_{(2)}, h_{(3)}^{(2)}\right) \# h_{(3)}^{(1)} S\left(h^{(2)}{ }_{(1)}\right)_{(3)}
\end{aligned}
$$

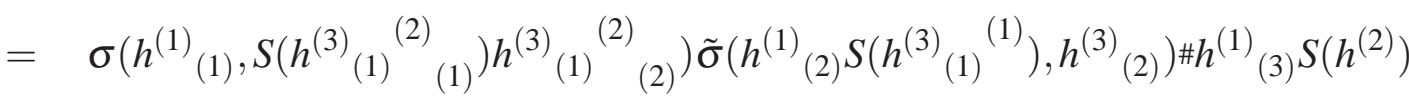

$$
\begin{aligned}
& =\tilde{\sigma}\left(s_{L}\left(\pi_{L}\left(h^{(1)}{ }_{(1)} s_{R}\left(\pi_{R}\left(h^{(3)}{ }_{(1)}{ }^{(2)}\right)\right)\right)\right) h^{(1)}{ }_{(2)} S\left(h^{(3)}{ }_{(1)}{ }^{(1)}\right), h^{(3)}{ }_{(2)}\right) \# h^{(1)}{ }_{(3)} S\left(h^{(2)}\right) \\
& =\tilde{\sigma}\left(h^{(1)}{ }_{(1)} S\left(h^{(3)}{ }_{(1)}{ }^{(1)} s_{R}\left(\pi_{R}\left(h^{(3)}{ }_{(1)}{ }^{(2)}\right)\right)\right), h^{(3)}{ }_{(2)}\right) \# h^{(1)}{ }_{(2)} S\left(h^{(2)}\right) \\
& =\tilde{\sigma}\left(h^{(1)}{ }_{(1)} S\left(h^{(3)}{ }_{(1)}\right), h_{(2)}^{(3)}\right) \# h_{(2)}^{(1)} S\left(h^{(2)}\right) \\
& =\tilde{\sigma}\left(h^{(1)}{ }_{(1)} S\left(h^{(2)}{ }_{(1)}\right), h^{(2)}{ }_{(2)}\right) \# h^{(1)}{ }_{(2)}{ }^{(1)} S\left(h^{(1)}{ }_{(2)}{ }^{(2)}\right) \\
& =\tilde{\sigma}\left(t_{L}\left(\pi_{L}\left(h^{(1)}{ }_{(2)}\right)\right) h^{(1)}{ }_{(1)} S\left(h^{(2)}{ }_{(1)}\right), h^{(2)}{ }_{(2)}\right) \# 1_{H} \\
& =\tilde{\sigma}\left(h_{(1)}{ }^{(1)} S\left(h_{(1)}{ }^{(2)}\right), h_{(2)}\right) \# 1_{H}=\tilde{\sigma}\left(1_{H}, h\right) \# 1_{H}=\eta_{L}\left(\pi_{L}(h)\right) \text {, }
\end{aligned}
$$

where the second equality follows by Lemma 4.10 (b), condition (c) in Definition 4.8 , condition (a) in Definition 4.1, condition (a) in Definition 4.8 and the counit property of $\pi_{L}$. The third equality follows by the anti-comultiplicativity of $S$ and (2.7). The fourth one follows by the antipode axiom (2.9), the fact that the domain of $\sigma$ is $H \otimes_{L^{o p}} H$ (i.e. $\sigma\left(h t_{L}(l), k\right)=\sigma\left(h, t_{L}(l) k\right)$ for $\left.h, k \in H, l \in L\right)$, the normalisation of cocycles (condition (c) in Definition 4.2) and the left $L$-linearity of $\tilde{\sigma}$ in the first argument (condition (a) in Definition 4.8). In the fifth step the Hopf algebroid identity $\pi_{L}\left(h s_{R}(r)\right)=\pi_{L}\left(h S\left(s_{R}(r)\right)\right)$, implying $s_{L}\left(\pi_{L}\left(h_{(1)} s_{R}(r)\right)\right) h_{(2)}=h S\left(s_{R}(r)\right)$, for $h \in H$ and $r \in R$, has been used together with the anti-multiplicativity of $S$. The sixth and seventh equalities follow by the coassociativity and $\mathscr{H}_{L}$-colinearity of $\gamma_{R}$ and the counit property of $\pi_{R}$. The eighth equality follows by the antipode axiom (2.9) and the right $L$-linearity of $\tilde{\sigma}$. The ninth one follows by axiom (2.7) and the counit property of $\pi_{L}$. The penultimate equality follows by axiom (2.9) and the fact that the domain of $\tilde{\sigma}$ is $H \otimes_{L} H$ (i.e. $\tilde{\sigma}\left(h s_{L}(l), k\right)=\tilde{\sigma}\left(h, s_{L}(l) k\right)$ for $\left.h, k \in H, l \in L\right)$. The last equality follows by Lemma 4.9. Note that all expressions in the above computation are well defined. In order to see that, recall that the restriction of $H \otimes_{R} \pi_{R}$ to the Takeuchi product $H \times{ }_{R} H$ is right $L$-linear (making the expression in the fifth line meaningful) and, similarly, the restriction of $H \otimes_{L} \pi_{L}$ to $H \times_{L} H$ is right $R$-linear (making the expression in the penultimate line meaningful).

The final aim of this section is to prove that any cleft extension is necessarily isomorphic to a crossed product with an invertible cocycle.

Theorem 4.12. If $B \subseteq A$ is an $\mathscr{H}$-cleft extension, then there exists an invertible cocycle $\sigma$ and a left B-module right $\mathscr{H}$-comodule algebra isomorphism $A \rightarrow B{ }_{\sigma} \mathscr{H}_{L}$.

Proof. For an $\mathscr{H}$-cleft extension $B \subseteq A$ the cleaving map $j$ takes the unit element of $H$ to an invertible element of $B$ (with the inverse $j^{c}\left(1_{H}\right)$ ). Thus, without the loss of generality, we can assume that a cleaving map $j$ is normalised, i.e. $j\left(1_{H}\right)=1_{A}=j^{\mathrm{C}}\left(1_{H}\right)$. By Theorem 3.12 $A$ is isomorphic to $B \otimes_{L} H$ as a left $B$-module and a right $\mathscr{H}$-comodule. We can use this isomorphism to induce a comodule algebra structure on $B \otimes_{L} H$. By Theorem 4.4 
the induced algebra structure is necessarily a crossed product $B{ }^{\#} \sigma \mathscr{H}_{L}$. In view of the definitions of the map $\kappa$ and its inverse in the proof of Theorem $3.12(1) \Rightarrow(2)(b)$, the measuring and cocycle can be read off equations (4.8) and (4.10), respectively, and come out as

$$
h \cdot b=j\left(h^{(1)}\right) b j^{\mathrm{c}}\left(h^{(2)}\right), \quad \sigma(h, k)=j\left(h^{(1)}\right) j\left(k^{(1)}\right) j^{\mathrm{c}}\left(h^{(2)} k^{(2)}\right) .
$$

We only need to prove that the cocycle $\sigma$ is invertible. Define

$$
\tilde{\sigma}: H \underset{L}{\otimes} H \rightarrow B, \quad h \underset{L}{\otimes} k \mapsto j\left(h^{(1)} k^{(1)}\right) j^{\complement}\left(k^{(2)}\right) j^{\complement}\left(h^{(2)}\right) .
$$

The map (4.19) is well defined by (3.3) and (3.4), on one hand, and by (3.4), (3.8) and the property that the range of the coproduct of a right bialgebroid is in the Takeuchi product, on the other hand. The proof that the range of $\tilde{\sigma}$ is in $B$ and that $\tilde{\sigma}$ is the inverse of the cocycle $\sigma$ are done by a routine calculation and are left to the reader.

Combining Theorem 4.7 with Theorem 4.12, we can fully describe the relationship between different cleaving maps for the same cleft extension.

Corollary 4.13. Let $\mathscr{H}$ be a Hopf algebroid and $B \subseteq A$ an $\mathscr{H}$-cleft extension with a (nonnecessarily unital) cleaving map $j: H \rightarrow A$. Then a map $j^{\prime}: H \rightarrow A$ is a cleaving map if and only if there exists a convolution invertible $L-L$ bilinear map $\chi: H \rightarrow B$, such that

$$
j^{\prime}(h)=\chi\left(h_{(1)}\right) j\left(h_{(2)}\right), \quad \text { for all } h \in H .
$$

Proof. If $j$ is a cleaving map and $\chi \in \operatorname{Hom}_{L, L}(H, A)$ is convolution invertible, then (4.20) obviously defines a cleaving map. In order to prove the converse claim, suppose first that both $j$ and $j^{\prime}$ are normalised as in the proof of Theorem 4.12, By Theorem 4.12, the crossed products corresponding to $j$ and $j^{\prime}$ are isomorphic to $A$ via left $B$-module right $\mathscr{H}$-comodule algebra maps, hence they are equivalent to each other. The isomorphism, obtained from combining the maps $v$ (for $j^{\prime}$ ) with $\kappa$ (for $j$ ) in the proof of Theorem $3.12(1) \Rightarrow(2)(b)$, explicitly comes out as $\Phi: b \otimes_{L} h \mapsto b j^{\prime}\left(h_{(1)}{ }^{(1)}\right) j^{c}\left(h_{(1)}{ }^{(2)}\right) \otimes_{L} h_{(2)}$. Then, by Theorem 4.7 the existence of $\Phi$ is equivalent to the existence of a normalised convolution invertible map $\chi \in \operatorname{Hom}_{L, L}(H, B), \chi(h)=j^{\prime}\left(h^{(1)}\right) j^{\mathrm{c}}\left(h^{(2)}\right)$. Using the right $\mathscr{H}_{L}$-colinearity of $\gamma_{R}$ and the fact that $j^{\mathrm{c}}$ is a left convolution inverse of $j$, one finds, for all $h \in H, \chi\left(h_{(1)}\right) j\left(h_{(2)}\right)=j^{\prime}(h)$, i.e. equation (4.20) holds. Allowing for $j, j^{\prime}$ to be non-unital is equivalent to not requiring that $\chi$ be normalised.

\section{The Relative Chern-Galois Character FOR $\mathscr{H}$-CLEFT EXTEnsions}

The aim of this section is to give a complete description of strong connections in a cleft extension $B \subseteq A$ of a Hopf algebroid $\mathscr{H}=\left(\mathscr{H}_{L}, \mathscr{H}_{R}, S\right.$ ) (over rings $L$ and $R$ ) and to find criteria for the existence and independence on the strong connection of the corresponding relative Chern-Galois characters introduced and computed in [11].

Begin with a right $\mathscr{H}$-extension $B \subseteq A$ and suppose that $T$ is a subalgebra of $B$. Then $A$ is called an $\left(\mathscr{H}_{R}, T\right)$-projective left $B$-module provided there exists a left $B$-linear, right $\mathscr{H}_{R^{-}}$ colinear section $\alpha_{T}$ of the multiplication map $B \otimes_{T} A \rightarrow A$. To consider the most general case possible, we make no assumptions on a ring $T$ (but, possibly, the most natural choice for $T$ is the base algebra $L$ ).

Lemma 5.1. Let $\mathscr{H}$ be a Hopf algebroid and $B \subseteq A$ an $\mathscr{H}$-cleft extension. Then $A$ is an $\left(\mathscr{H}_{R}, L\right)$-projective left $B$-module. 
Proof. The map $\tilde{\alpha}_{L}: B \otimes_{L} H \rightarrow B \otimes_{L} B \otimes_{L} H, b \otimes_{L} h \mapsto b \otimes_{L} 1_{B} \otimes_{L} h$ is a left $B$-linear right $\mathscr{H}$-colinear splitting of the product map $b \otimes_{L} b^{\prime} \otimes_{L} h \mapsto b b^{\prime} \otimes_{L} h$. By Theorem 3.12 $A \simeq B \otimes_{L} H$ as left $B$-modules and right $\mathscr{H}$-comodules, hence there is a corresponding splitting $\alpha_{L}$ of the $B$-product map in $A$. Explicitly,

$$
\alpha_{L}=\left(B \underset{L}{\otimes} \kappa^{-1}\right) \circ \tilde{\alpha}_{L} \circ \kappa, \quad a \mapsto a_{[0]}^{[0]} j^{\mathrm{c}}\left(a_{[0]}^{[1]}\right) \underset{L}{\otimes} j\left(a_{[1]}\right)=a^{[0]} j^{\mathrm{c}}\left(a^{[1]}(1)\right) \underset{L}{\otimes} j\left(a^{[1]}{ }_{(2)}\right),
$$

where $\kappa$ is the isomorphism (3.12) in the proof of Theorem 3.12 and $j$ is a cleaving map with the convolution inverse $j^{\mathrm{C}}$.

Any right $\mathscr{H}$-comodule algebra $A$ with $\mathscr{H}_{R}$-coaction $\rho^{A}: a \mapsto a^{[0]} \otimes_{R} a^{[1]}$ and $\mathscr{H}_{L}$-coaction $\lambda^{A}: a \mapsto a_{[0]} \otimes_{L} a_{[1]}$ gives rise to an entwining map (over $R$ ) $\psi: H \otimes_{R} A \rightarrow A \otimes_{R} H, h \otimes_{R}$ $a \mapsto a^{[0]} \otimes_{R} h a^{[1]}$. The map $\psi$ is bijective, provided the antipode $S$ is bijective (cf. [9, Lemma 4.1]), and then the corresponding left $\mathscr{H}_{R}$-coaction on $A$ is

$$
{ }^{A} \rho: A \rightarrow H \underset{R}{\otimes} A, \quad a \mapsto S^{-1}\left(a_{[1]}\right) \otimes_{R} a_{[0]}
$$

(compare with (2.20) ). Thus, following [11, Definition 3.4], if $B \subseteq A$ is a right $\mathscr{H}$-extension

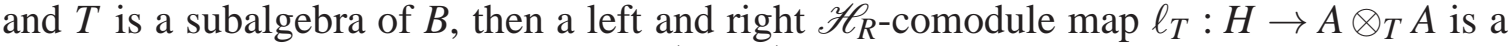
strong $T$-connection provided that $\widetilde{\operatorname{can}}_{T}\left(\ell_{T}(h)\right)=1_{A} \otimes_{R} h$, for all $h \in H$, where the map

$$
\widetilde{\operatorname{can}}_{T}: A \underset{T}{\otimes A} \rightarrow A \underset{R}{\otimes_{1} H,} \quad a \otimes_{T} a^{\prime} \mapsto a a^{\prime[0]} \otimes_{R} a^{\prime[1]},
$$

is well defined by the left $T$-linearity of $\rho^{A}$. The $\mathscr{H}_{R}$-coactions in $A \otimes_{T} A$ are $A \otimes_{T} \rho^{A}$ and ${ }^{A} \rho \otimes_{T} A$, with ${ }^{A} \rho$ given in (5.1).

The first observation is that a cleft extension comes equipped with a strong $L$-connection.

Theorem 5.2. Let $\mathscr{H}$ be a Hopf algebroid with a bijective antipode and $B \subseteq A$ an $\mathscr{H}$-cleft extension with a cleaving map $j$. Then the map

$$
\ell_{L}: H \rightarrow A \underset{L}{\otimes} A, \quad h \mapsto j^{c}\left(h_{(1)}\right) \underset{L}{\otimes} j\left(h_{(2)}\right),
$$

is a strong L-connection.

Proof. By Theorem 3.12, $B \subseteq A$ is a Galois $\mathscr{H}_{R}$-extension, which is $\left(\mathscr{H}_{R}, L\right)$-projective by Lemma 5.1. Thus the existence of a strong connection follows by [11, Theorem 3.7]. Using the explicit forms of the inverse of the canonical $\mathscr{H}_{R}$-Galois map in the proof of Theorem 3.12 and of $\alpha_{L}$ in the proof of Lemma 5.1, following the proof of [11, Theorem 3.7] one arrives at the form of a strong $L$-connection in (5.3).

The full classification of strong $T$-connections in a cleft extension is described in forthcoming Theorem 5.5. Its proof relies on two lemmata:

Lemma 5.3. For any Hopf algebroid $\mathscr{H}$, the following statements hold.

(1) The forgetful functor $\mathbf{M}^{\mathscr{H}} \rightarrow \mathbf{M}_{L}$ possesses a right adjoint $(-) \otimes_{L} H$.

(2) The forgetful functor ${ }^{\mathscr{H}} \mathbf{M} \rightarrow{ }_{L} \mathbf{M}$ possesses a right adjoint $H \otimes_{L}(-)$.

Proof. (1) The unit of the adjunction is given by the $\mathscr{H}_{L}$-coaction $M \rightarrow M \otimes_{L} H$, for any right $\mathscr{H}$-comodule $M$. It is an $\mathscr{H}$-comodule map by definition. The counit is given by $N \otimes_{L} \pi_{L}: N \otimes_{L} H \rightarrow N$, for any right $L$-module $N$. Part (2) is proven symmetrically.

Lemma 5.4. Let $\mathscr{H}$ be a Hopf algebroid with a bijective antipode and $B \subseteq A$ an $\mathscr{H}$-cleft extension. Then the obvious inclusion $\operatorname{Hom}^{\mathscr{H}, \mathscr{H}}\left(H, A \otimes_{T} A\right) \hookrightarrow \operatorname{Hom}^{\mathscr{H}_{R}, \mathscr{H}_{R}}\left(H, A \otimes_{T} A\right)$ is an isomorphism. 
Proof. We need to show that any $f \in \operatorname{Hom}^{\mathscr{H}_{R}, \mathscr{H}_{R}}\left(H, A \otimes_{T} A\right)$ is also left and right $\mathscr{H}_{L^{-}}$ colinear. Indeed, in terms of a cleaving map $j$ and its convolution inverse $j^{c}$, for any $h \in H$,

$$
\begin{aligned}
f(h) & =f\left(h^{(1)} s_{R}\left(\pi_{R}\left(h^{(2)}\right)\right)\right)=f\left(h^{(1)}\right) \eta_{R}\left(\pi_{R}\left(h^{(2)}\right)\right)=f\left(h^{(1)}\right) j^{\mathrm{C}}\left(h^{(2)}(1)\right) j\left(h^{(2)}{ }_{(2)}\right) \\
& =f\left(h_{(1)}^{(1)}\right) j^{\mathrm{C}}\left(h_{(1)}{ }^{(2)}\right) j\left(h_{(2)}\right)=f\left(h_{(1)}\right)^{[0]} j^{\mathrm{C}}\left(f\left(h_{(1)}\right)^{[1]}\right) j\left(h_{(2)}\right) .
\end{aligned}
$$

The first equality follows by the counit property of $\pi_{R}$ and the second one follows by the right $R$-linearity of $f$. In the third equality we used that $j^{c}$ is left convolution inverse of $j$, i.e. (3.6). The penultimate equality is a consequence of the right $\mathscr{H}_{L}$-colinearity of $\gamma_{R}$ and the last equality follows by the right $\mathscr{H}_{R}$-colinearity of $f$. By Lemma 3.10, the left $B$-linearity of the right $\mathscr{H}_{L}$-coaction on $A$ and the right $\mathscr{H}_{L}$-colinearity of $j$, this proves that $f$ is right $\mathscr{H}_{L}$-colinear. Left $\mathscr{H}_{L}$-colinearity of $f$ is proven symmetrically.

Theorem 5.5. Let $\mathscr{H}$ be a Hopf algebroid with a bijective antipode and $B \subseteq A$ an $\mathscr{H}$-cleft extension, and let $T$ be a subalgebra of $B$. Write $\mu_{B}$ for the multiplication map $B \otimes_{T} B \rightarrow B$. Strong $T$-connections in $B \subseteq A$ are in bijective correspondence with $L$ - $L$ bilinear maps $f: H \rightarrow B \otimes_{T} B$ such that $\mu_{B} \circ f=\eta_{L} \circ \pi_{L}$.

Proof. Let $j$ be a cleaving map and $j^{c}$ its convolution inverse. By Lemma 5.4, Theorem 3.12 and Corollary 3.13 and finally Lemma 5.3, there is a chain of isomorphisms

$\operatorname{Hom}^{\mathscr{H}_{R}, \mathscr{H}_{R}}(H, A \underset{T}{\otimes A}) \simeq \operatorname{Hom}^{\mathscr{H}, \mathscr{H}}(H, A \underset{T}{\otimes A}) \simeq \operatorname{Hom}^{\mathscr{H}, \mathscr{H}}\left(H, H \underset{L}{\otimes}{\underset{T}{\otimes}}_{L} B \underset{L}{\otimes} H\right) \simeq \operatorname{Hom}_{L, L}(H, B \underset{T}{\otimes B})$.

In view of the explicit form of the isomorphism $\kappa: A \rightarrow B \otimes_{L} H$ in (3.12) and its left-handed version (3.13), we thus obtain:

$$
\operatorname{Hom}^{\mathscr{H}_{R}, \mathscr{H}_{R}}(H, A \underset{T}{\otimes} A) \ni \ell_{T} \mapsto\left[f: h \mapsto j\left(h^{(1)}\right) \ell_{T}\left(h^{(2)}\right) j^{\complement}\left(h^{(3)}\right)\right],
$$

and its inverse

$$
\operatorname{Hom}_{L, L}(H, B \underset{T}{\otimes} B) \ni f \mapsto\left[\ell_{T}: h \mapsto j^{\mathrm{c}}\left(h_{(1)}\right) f\left(h_{(2)}\right) j\left(h_{(3)}\right)\right] .
$$

If $\ell_{T}$ is a strong $T$-connection, then $\mu_{A} \circ \ell_{T}=\eta_{R} \circ \pi_{R}$, where $\mu_{A}: A \otimes_{T} A \rightarrow A$ is the product map in $A$. This implies that for the corresponding $f$ in (5.4), $\mu_{B} \circ f=\eta_{L} \circ \pi_{L}$. Conversely, suppose that $f$ has this property, write $f(h)=h^{\{1\}} \otimes_{T} h^{\{2\}}$ for all $h \in H$, and compute

$$
\begin{aligned}
& \widetilde{\operatorname{can}}_{T}\left(\ell_{T}(h)\right)=j^{\mathrm{c}}\left(h_{(1)}\right) h_{(2)}{ }^{\{1\}} h_{(2)}\{2\} j\left(h_{(3)}\right)^{[0]}{\underset{R}{\otimes}}_{j} j\left(h_{(3)}\right)^{[1]} \\
& =j^{\mathrm{c}}\left(h_{(1)}\right) \eta_{L}\left(\pi_{L}\left(h_{(2)}\right)\right) j\left(h_{(3)}{ }^{(1)}\right) \otimes_{R} h_{(3)}{ }^{(2)} \\
& =j^{\complement}\left(h_{(1)}\right) j\left(h_{(2)}{ }^{(1)}\right){\underset{R}{B} h_{(2)}}^{(2)}=j^{\complement}\left(h^{(1)}{ }_{(1)}\right) j\left(h^{(1)}{ }_{(2)}\right) \underset{R}{\otimes} h^{(2)} \\
& =\eta_{R}\left(\pi_{R}\left(h^{(1)}\right)\right) \underset{R}{\otimes} h^{(2)}=1_{A} \otimes_{R} h,
\end{aligned}
$$

where the first equality follows by (5.2) and the fact that the range of $f$ is in $B \otimes_{T} B$. The second equality follows by the hypothesis $\mu_{B} \circ f=\eta_{L} \circ \pi_{L}$ and the right $\mathscr{H}$-colinearity of $j$. The third one follows by the left $L$-linearity of $j$ (i.e. (3.3) ) and the counit property of $\pi_{L}$. The fourth equality follows by the left $\mathscr{H}_{L}$-colinearity of $\gamma_{R}$. In the penultimate step we used that $j^{c}$ is a left convolution inverse of $j$, i.e. (3.6). This means that $\ell_{T}$ given in (5.5) is a strong $T$-connection and completes the proof of the theorem.

Take a bijective entwining structure $(A, C, \psi)_{R}$ over a non-commutative base algebra $R$ and consider a $T$-flat entwined extension $B \subseteq A$ in the sense of [11, Definition 5.2] ( $T$ is a subalgebra of $B$ ). Given a strong $T$-connection in $B \subseteq A$, one constructs a family of maps of Abelian groups from the Grothendieck group of $C$-comodules to the even $T$-relative cyclic 
homology groups of $B$ (cf. [11, Theorem 5.4]). This family of maps is termed the T-relative Chern-Galois character. Comodule algebras for Hopf algebroids (with bijective antipodes) provide examples of (bijective) entwining structures over non-commutative bases, hence the general theory worked out in [11] can be applied to such algebras. In particular, the components of the $T$-relative Chern-Galois characters, corresponding to strong $T$-connections in Theorem 5.5 for a $T$-flat cleft extension of a Hopf algebroid with bijective antipode, have been computed in [11, Example 5.6].

It is important to note, however, that the $T$-relative Chern-Galois character, a priori, depends on the choice of a strong $T$-connection. Its independence is proven in [11, Theorem 5.14], under the assumption that the $T$-flat entwined extension $B \subseteq A$ enjoys the following properties:

(a) $A$ is a locally projective right $T$-module;

(b) the extension $B \subseteq A$ splits as a $B-T$ bimodule.

In the remainder of this section we analyse the meaning of these conditions and of the $T$-flatness in the case of cleft Hopf algebroid extensions. In this way we find sufficient conditions for the existence and the strong-connection-independence of the relative ChernGalois character computed in [11, Example 5.6].

Definition 5.6. Let $B \subseteq A$ be a right extension of a Hopf algebroid $\mathscr{H}$ with a bijective antipode and let $T$ be a subalgebra of $B$. View $A$ as a left $\mathscr{H}_{R}$-comodule with coaction (5.1) and $\mathscr{H}_{L}$-coaction $a \mapsto S^{-1}\left(a^{[1]}\right) \otimes_{L} a^{[0]}$. A left total $T$-integral is a left $\mathscr{H}$-colinear map $\vartheta: H \rightarrow A$ such that $\vartheta(H) \subseteq A^{T}:=\{a \in A \mid t a=a t \quad \forall t \in T\}$ and $\vartheta\left(1_{H}\right)=1_{A}$.

For example, the convolution inverse of a normalised cleaving map is a left total $k$ integral by Lemma 3.8. By arguments similar to those used to prove Lemma 3.10, any left total $T$-integral $\vartheta$ determines a $B$ - $T$ bilinear section of the extension $B \subseteq A$,

$$
a \mapsto a^{[0]} \vartheta\left(a^{[1]}\right) .
$$

The next proposition shows that, for a cleft extension of a Hopf algebroid with a bijective antipode, this is a one-to-one correspondence.

Note that if $B \subseteq A$ is a cleft extension by a Hopf algebroid $\mathscr{H}$ with a bijective antipode, then similarly to the proof of Lemma 5.4, any morphism $f \in \operatorname{Hom}^{\mathscr{H}_{R},-}(H, A)$ satisfies $f(h)=j^{\mathrm{C}}\left(h_{(1)}\right) j\left(S^{-1}\left(f\left(h_{(2)}\right)_{[1]}\right)\right) f\left(h_{(2)}\right)_{[0]}$, for any $h \in H$, where $j$ is a cleaving map with convolution inverse $j^{\mathrm{c}}$. Hence $f$ is also left $\mathscr{H}_{L}$-colinear, with respect to the $\mathscr{H}_{L}$-coaction $a \mapsto S^{-1}\left(a^{[1]}\right) \otimes_{L} a^{[0]}$ on $A$. In particular, in this situation a left total $T$-integral is the same as a left $\mathscr{H}_{R}$-colinear map $\vartheta: H \rightarrow A$ such that $\vartheta(H) \subseteq A^{T}$ and $\vartheta\left(1_{H}\right)=1_{A}$.

Proposition 5.7. Let $B \subseteq A$ be a cleft extension of a Hopf algebroid $\mathscr{H}$ with a bijective antipode, and let $T$ be a subalgebra of $B$. Then $B$-T bilinear sections of the extension $B \subseteq A$ are in bijective correspondence with left total $T$-integrals in $B \subseteq A$.

Proof. Let $j$ be a cleaving map for $B \subseteq A$. In terms of $j$ we construct the inverse of the map associating to a left total $T$-integral $\vartheta$ the section (5.6). To a $B-T$ bilinear section $\varphi$, associate the map

$$
\vartheta: H \rightarrow A, \quad h \mapsto j^{\mathrm{C}}\left(h_{(1)}\right) \varphi\left(j\left(h_{(2)}\right)\right) .
$$

Since $j\left(1_{H}\right)$ is an element of $B, \vartheta\left(1_{H}\right)=j^{\mathrm{c}}\left(1_{H}\right) j\left(1_{H}\right) \varphi\left(1_{A}\right)=1_{A}$. The left $\mathscr{H}$-colinearity of $\vartheta$ follows by the left $\mathscr{H}$-colinearity of $\gamma_{L}$ and $j^{c}$, and the fact that the range of $\varphi$ is equal 
to $B$. It remains to check that the range of $\vartheta$ is in $A^{T}$. Note that by its left $B$-linearity, $\varphi$ is determined by the left $L$-linear map $\varphi \circ j: H \rightarrow B$. Indeed, for all $a \in A$,

$\varphi(a)=\varphi\left(a^{[0]} j^{\complement}\left(a^{[1]}(1)\right) j\left(a^{[1]}(2)\right)\right)=\varphi\left(a_{[0]}^{[0]} j^{\complement}\left(a_{[0]}{ }^{[1]}\right) j\left(a_{[1]}\right)\right)=a_{[0]}[0] j^{\complement}\left(a_{[0]}^{[1]}\right) \varphi\left(j\left(a_{[1]}\right)\right)$, where in the last equality Lemma 3.10 has been used. Hence the right $T$-linearity of $\varphi$ is equivalent to

$$
a^{[0]} t j^{\mathrm{C}}\left(a^{[1]}{ }_{(1)}\right) \varphi\left(j\left(a^{[1]}{ }_{(2)}\right)\right)=\varphi(a) t, \quad \text { for all } a \in A \text { and } t \in T .
$$

Take any $h \in H$ and apply (5.8) to $a=j(h)$. By the right $\mathscr{H}_{R}$-colinearity of $j$,

$$
j\left(h^{(1)}\right) t j^{\mathrm{C}}\left(h^{(2)}{ }_{(1)}\right) \varphi\left(j\left(h^{(2)}{ }_{(2)}\right)\right)=\varphi(j(h)) t .
$$

Hence

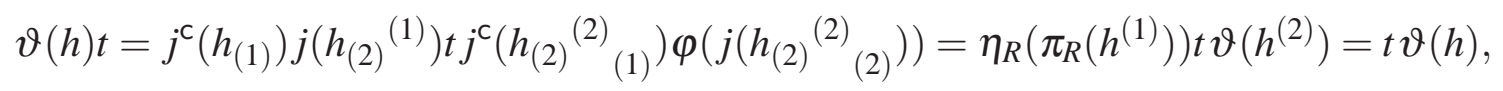

where the second equality follows by the Hopf algebroid axiom (2.7) and the last one follows by the fact that the elements of $B$ (and hence, in particular, the elements of $T$ ) commute with $\eta_{R}(r)$, for $r \in R$, and by the left $R$-linearity of $\vartheta$. It is checked by a routine computation that the map, associating to a $B-T$ bilinear section $\varphi$ of the inclusion $B \subseteq A$ the left total $T$-integral (5.7), is the inverse of the map, associating to the left total $T$-integral $\vartheta$ the $B-T$ bilinear section (5.6).

For a cleft extension $B \subseteq A$ of a Hopf algebroid $\mathscr{H}$ with a bijective antipode, consider the $B-B$ bilinear map,

$$
A \rightarrow A \otimes_{R} H, \quad a \mapsto a^{[0]} \otimes_{R} a^{[1]}-a \otimes_{R} 1_{H},
$$

where $A \otimes_{R} H$ is a $B-B$ bimodule via the first tensorand. For any subalgebra $T$ of $B$ this map projects to the map $v_{T}: A /[A, T] \rightarrow A /[A, T] \otimes_{R} H$, where $[A, T]=\left\{\sum_{k}\left(a_{k} t_{k}-t_{k} a_{k}\right) \mid a_{k} \in\right.$ $\left.A, t_{k} \in T\right\}$ is a right $R$-submodule of $A$. Following [11, Definition 5.2], the extension $B \subseteq A$ is said to be $T$-flat if $B$ and $A$ are flat left and right $T$-modules and the obvious map

$$
B /[B, T] \rightarrow \text { ker } v_{T}, \quad[b]_{B} \mapsto[b]_{A},
$$

(where []$_{B}$ denotes the equivalence class in $B /[B, T]$ and []$_{A}$ denotes the equivalence class in $A /[A, T])$ is an isomorphism.

Proposition 5.8. Let $\mathscr{H}$ be a Hopf algebroid with a bijective antipode. A cleft $\mathscr{H}$-extension $B \subseteq A$ which splits as a $B$-T bimodule for some subalgebra $T$ of $B$, is $T$-flat if and only if $A$ is a flat left and right $T$-module.

Proof. Since a direct summand of a flat module is flat, it suffices to prove that the existence of a $B-T$ bimodule splitting of the inclusion, i.e. the existence of a left total $T$ integral, implies that the map (5.9) is an isomorphism. In order to prove injectivity of (5.9), choose $b \in B$ such that $[b]_{A}=0$. This means the existence of finite sets $\left\{a_{k}\right\}$ in $A$ and $\left\{t_{k}\right\}$ in $T$ such that $b=\sum_{k}\left(a_{k} t_{k}-t_{k} a_{k}\right)$. Applying a $B-T$ bilinear section $\varphi$ of the extension $B \subseteq A$ to this identity, we obtain $b=\sum_{k}\left(\varphi\left(a_{k}\right) t_{k}-t_{k} \varphi\left(a_{k}\right)\right)$, hence $[b]_{B}=0$. In order to prove the surjectivity of the map (5.9), choose $a \in A$ such that $v_{T}\left([a]_{A}\right)=0$. This means the existence of finite sets $\left\{a_{k}\right\}$ in $A,\left\{h_{k}\right\}$ in $H$ and $\left\{t_{k}\right\}$ in $T$ such that

$$
a^{[0]} \otimes_{R} a^{[1]}-a_{R}^{\otimes} 1_{H}=\sum_{k}\left(a_{k} t_{k} \otimes_{R} h_{k}-t_{k} a_{k} \otimes_{R} h_{k}\right) .
$$


By Proposition 5.7, there is a left total $T$-integral $\vartheta$ in $B \subseteq A$. Apply $\mu_{A} \circ\left(A \otimes_{R} \vartheta\right)$ to (5.10) to obtain

$$
a^{[0]} \vartheta\left(a^{[1]}\right)-a=\sum_{k}\left(a_{k} \vartheta\left(h_{k}\right) t_{k}-t_{k} a_{k} \vartheta\left(h_{k}\right)\right) \text {. }
$$

This proves that $[a]_{A}=\left[a^{[0]} \vartheta\left(a^{[1]}\right)\right]_{A}$. Since $a^{[0]} \vartheta\left(a^{[1]}\right)$ is an element of $B,[a]_{A}$ belongs to the image of the map (5.9).

Combining Proposition 5.7 and Proposition 5.8 with [11, Theorem 5.14] we obtain

Corollary 5.9. Let $B \subseteq A$ be a cleft extension of a Hopf algebroid with a bijective antipode and let $T$ be a subalgebra of $B$. Assume that:

(a) A is a flat left $T$-module and a locally projective right $T$-module;

(b) there exists a left total $T$-integral for the extension $B \subseteq A$;

(c) there exists a strong T-connection.

Then there exists a T-relative Chern-Galois character, independent on the choice of the strong $T$-connection in (c).

We close the section with some examples of Hopf algebroid cleft extensions, in which there exist (strong-connection-independent) relative Chern-Galois characters.

Example 5.10. Let $B \subseteq A$ be a cleft extension of a Hopf algebroid with a bijective antipode and $T$ a separable $k$-subalgebra of $B$. In light of [11, Proposition 3.2 (1)], since $B \subseteq A$ splits as a left $B$-module by Lemma 3.11 , it splits as a $B-T$ bimodule. The corresponding total $T$-integral $\vartheta$ in $B \subseteq A$ is

$$
\vartheta(h)=\sum_{i} e_{i} j^{\mathrm{c}}(h) j\left(1_{H}\right) e^{i}
$$

where $\sum_{i} e_{i} \otimes_{k} e^{i} \in T \otimes_{k} T$ is a separability idempotent. Therefore, if $A$ is a flat left $T$ module and a locally projective right $T$-module and there exists a strong $T$-connection $\ell_{T}$, then there exists a corresponding $T$-relative Chern-Galois character which is independent of $\ell_{T}$.

Example 5.11. The base algebra $R$ of a Hopf algebroid $\mathscr{H}$ is a right $\mathscr{H}$-comodule algebra with $\mathscr{H}_{R}$-coaction $r \mapsto 1_{R} \otimes_{R} s_{R}(r)$ and $\mathscr{H}_{L}$-coaction $r \mapsto 1_{R} \otimes_{L} s_{R}(r)$. It follows by [8, Theorem 3.2] that a Hopf algebroid $\mathscr{H}$ with a bijective antipode is coseparable (as an $L$ - or, equivalently, as an $R$-coring) if and only if there exists a left total $k$-integral $\lambda$ for the $\mathscr{H}$ extension $I \subseteq R$, where $I$ is the $\mathscr{H}_{R}$-coinvariant subalgebra of $R, I=\left\{r \in R \mid s_{R}(r)=t_{R}(r)\right\}$. Note that if $\mathscr{H}$ is a coseparable Hopf algebroid, then any right $\mathscr{H}$-extension $B \subseteq A$ is split as a $B-B$ bimodule by the map

$$
A \rightarrow B, \quad a \mapsto a^{[0]} \eta_{R}\left(\lambda\left(a^{[1]}\right)\right) .
$$

Let $B \subseteq A$ be a cleft extension of a coseparable Hopf algebroid with a bijective antipode and $T$ a subalgebra of $B$. Then there is a left total $T$-integral $\vartheta=\eta_{R} \circ \lambda$. If $A$ is a locally projective right $T$-module and a flat left $T$-module and there exists a strong $T$-connection $\ell_{T}$, then the corresponding $T$-relative Chern-Galois character is independent of $\ell_{T}$.

\section{APPENDIX A. WEAK CLEFT EXTENSIONS AND WEAK CROSSED PRODUCTS}

Many of the results described in Sections 3, 4 and 5 (e.g. Lemma 3.11, Corollary 3.14, Corollary 3.15 or Theorem 5.2, Corollary 5.9 remain valid if the right $\mathscr{H}$-extension $B \subseteq A$ is an $\mathscr{H}_{R}$-Galois extension but, instead of the normal basis condition Theorem 3.12 (2)(b), 
$A$ is only a direct summand of $B \otimes_{L} H$ as a left $B$-module and as a right $\mathscr{H}$-comodule. Such extensions can be studied along the same lines as in Sections 3, 4 and 5, In this appendix we present the results of such studies; we give no proofs as these are very similar to the proofs of corresponding results in preceding sections.

Motivated by the forthcoming analogue of Theorem 3.12 (Theorem A.2), we introduce the following weakening of Definition 3.5 .

Definition A.1. Let $\mathscr{H}$ be a Hopf algebroid. A right $\mathscr{H}$-extension $B \subseteq A$ is weak cleft if

(a) in addition to its canonical $R$-ring structure, $A$ possesses an $L$-ring structure and $B$ is an $L$-subring of $A$;

(b) there exists a left $L$-linear right $\mathscr{H}$-colinear morphism $j: H \rightarrow A$, with left convolution inverse $j^{\mathrm{w}}$, which is right $\mathscr{H}$-colinear in the sense of identities (3.8) and (3.9).

A map $j$, satisfying condition (b), is called a weak cleaving map.

Note that in the situation described in Definition A.1, the assumption that $j^{\mathrm{w}}$ satisfies (3.9) implies that the image of the map $A \rightarrow A, a \mapsto a^{[0]} j^{\mathrm{w}}\left(a^{[1]}\right)$ is contained in $B$. Hence a weak $\mathscr{H}$-cleft extension $B \subseteq A$ is split by the left $B$-linear map (3.11) after replacing $j^{c}$ with $j^{\mathrm{w}}$.

Theorem A.2. Let $\mathscr{H}$ be a Hopf algebroid and $B \subseteq A$ a right $\mathscr{H}$-extension. Then the following statements are equivalent:

(1) $B \subseteq A$ is a weak $\mathscr{H}$-cleft extension.

(2) (a) The extension $B \subseteq A$ is $\mathscr{H}_{R}$-Galois;

(b) $A$ is a direct summand of $B \otimes_{L} H$ as a left $B$-module and right $\mathscr{H}$-comodule.

In particular, Theorem A.2 implies that if $\mathscr{H}$ is projective as a left $L$-module then, for any weak cleft $\mathscr{H}$-extension $B \subseteq A, A$ is a faithfully flat left $B$-module.

Recall that in Section 4 we applied a (unnormalised) gauge transformation to a general cleaving map in order to normalise it as $j\left(1_{H}\right)=1_{B}=j^{\mathrm{c}}\left(1_{H}\right)$. However, in the case when $j$ possesses a left convolution inverse $j^{\mathrm{w}}$ only, there is no guarantee for $j\left(1_{H}\right)$ to be an invertible element of $B$. Hence it can not be gauge transformed to the unit element in general. The need to describe this more general situation leads to the following generalisations of Definitions 4.1 and 4.2 .

Definition A.3. Let $\mathscr{L}=(H, L, s, t, \gamma, \pi)$ be a left bialgebroid and $B$ an $L$-ring. $\mathscr{L}$ weakly measures $B$ if there exits a $k$-linear map, termed a weak measuring, $H \otimes_{k} B \rightarrow B, h \otimes_{k} b \mapsto$ $h \cdot b$ that satisfies properties (b) and (c) in Definition 4.1.

Definition A.4. Let $\mathscr{L}=(H, L, s, t, \gamma, \pi)$ be a left bialgebroid and $B$ an $L$-ring, weakly measured by $\mathscr{L}$. A $B$-valued weak 2-cocycle $\sigma$ on $\mathscr{L}$ is a $k$-linear map $H \otimes_{L^{o p}} H \rightarrow B$ (where the right and left $L^{o p}$-module structures on $H$ are given by right and left multiplication by $t(l)$, respectively) satisfying properties (a), (b) and (d) in Definition 4.2 and, in addition, for all $h, k \in H$,

$$
\sigma\left(h_{(1)}, k_{(1)}\right)\left(h_{(2)} k_{(2)} \cdot 1_{B}\right)=\sigma(h, k) .
$$

A weakly $\mathscr{L}$-measured $L$-ring $B$ is called a $\sigma$-twisted left $\mathscr{L}$-module if a weak 2 -cocycle $\sigma$ satisfies property (f) in Definition 4.2 and there exist elements $x$ and $\tilde{x}$ in $B$ such that, for all 
$b \in B$ and $h \in H$,

$$
\begin{array}{rll}
\tilde{x} x=1_{B} & \text { and } & x b \tilde{x}=1_{H} \cdot b, \\
\sigma\left(h \cdot 1_{B}\right) & \text { and } & \sigma\left(h, 1_{H}\right)=h \cdot x .
\end{array}
$$

It is easy to see that a $B$-valued 2-cocycle is also a weak 2-cocycle. If the $L$-ring $B$ is a $\sigma$-twisted $\mathscr{L}$-module for a 2-cocycle $\sigma$, then it is a $\sigma$-twisted $\mathscr{L}$-module also in the weaker sense of Definition A.4 with $x=1_{B}=\tilde{x}$.

Recall from [21, p. 39] that, for a non-unital ring $A$, an element $e \in A$ such that, for all $a \in A, e a=a e=a e^{2}$ is called a preunit. Proposition 4.3 can be extended to the case of weak cocycles as follows.

Proposition A.5. Let $\mathscr{L}=(H, L, s, t, \gamma, \pi)$ be a left bialgebroid and $B$ an L-ring, weakly measured by $\mathscr{L}$. Let $\sigma: H \otimes_{L^{o p}} H \rightarrow B$ be a map that satisfies properties $(a)$ and $(b)$ in Definition 4.2 and condition (A.1) in Definition A.4 Consider the k-module $B \otimes_{L} H$ in Proposition 4.3. and the following assertions.

(a) $B \otimes_{L} H$ is an associative (possibly non-unital) algebra with multiplication (4.1).

(b) There exists $\tilde{y} \in B$ such that $\tilde{y} \sigma\left(1_{H}, h\right)=h \cdot 1_{B}$, for all $h \in H$, and $\tilde{y} \otimes_{L} 1_{H}$ is a preunit for the algebra in part (a) (hence $A:=\left\{\left(b \otimes_{L} h\right)\left(\tilde{y} \otimes_{L} 1_{H}\right) \mid b \otimes_{L} h \in B \otimes_{L} H\right\}$ is a right $\mathscr{L}$-comodule algebra with coinvariant subalgebra $\left.\left\{\left(b \otimes_{L} 1_{H}\right)\left(\tilde{y} \otimes_{L} 1_{H}\right) \mid b \in B\right\}\right)$.

(c) The map $B \rightarrow A^{c o \mathscr{L}}, b \mapsto\left(b \tilde{y} \otimes_{L} 1_{H}\right)\left(\tilde{y} \otimes_{L} 1_{H}\right)$ is an algebra isomorphism.

These assertions hold if and only if $\sigma$ is a weak 2-cocycle and $B$ is a $\sigma$-twisted left $\mathscr{L}$ module. In this case $A$ is called a weak crossed product of $B$ with $\mathscr{L}$ with respect to the weak 2-cocycle $\sigma$.

Our next task is to characterise equivalent weak crossed products, in analogy with Theorem 4.7

Definition A.6. Let $\mathscr{L}=(H, L, s, t, \gamma, \pi)$ be a left bialgebroid and $B$ an $L$-ring. Weak crossed product algebras of $B$ with $\mathscr{L}$ are said to be equivalent if they are isomorphic via a left $B$-linear isomorphism of right $\mathscr{L}$-comodule algebras.

Note that a (left $B$-linear right $\mathscr{L}$-colinear) isomorphism of weak crossed product algebras of $B$ with $\mathscr{L}$ in Definition A.6 needs not extend to the (non-unital) algebra $B \otimes_{L} H$. The following lemma extends Corollary 4.5 .

Lemma A.7. Let $\mathscr{L}=(H, L, s, t, \gamma, \pi)$ be a left bialgebroid and $B$ an L-ring, weakly measured by $\mathscr{L}$. Let $\sigma$ be a weak 2-cocycle, such that $B$ is a $\sigma$-twisted left $\mathscr{L}$-module. Let $\chi$ and $\tilde{\chi}$ be morphisms in $\operatorname{Hom}_{L, L}(H, B)$ such that, for all $h \in H$,

$$
\begin{aligned}
& \tilde{\chi}\left(h_{(1)}\right) \chi\left(h_{(2)}\right)=h \cdot 1_{B} \quad \text { and } \\
& \tilde{\chi}\left(h_{(1)}\right) \chi\left(h_{(2)}\right) \tilde{\chi}\left(h_{(3)}\right)=\tilde{\chi}(h), \quad \chi\left(h_{(1)}\right) \tilde{\chi}\left(h_{(2)}\right) \chi\left(h_{(3)}\right)=\chi(h) .
\end{aligned}
$$

Then (4.12) defines a weak measuring of $\mathscr{L}$ on $B$ and (4.13) defines a weak 2-cocycle $\sigma^{\chi}$, such that $B$ is a $\sigma^{\chi}$-twisted left $\mathscr{L}$-module.

A pair $\chi, \tilde{\chi} \in \operatorname{Hom}_{L, L}(H, B)$, satisfying A.2 A.3), is called a gauge transformation of the weak crossed product of $B$ with $\mathscr{L}$. Gauge transformations form a groupoid, with multiplication, the convolution product $\diamond$ in the first component, and its opposite in the second one. The left unit of a gauge transformation $(\chi, \tilde{\chi})$ is $(\chi \diamond \tilde{\chi}, \chi \diamond \tilde{\chi})$ and its right unit is $(\tilde{\chi} \diamond \chi, \tilde{\chi} \diamond \chi)$. The inverse of $(\chi, \tilde{\chi})$ is $(\tilde{\chi}, \chi)$. 
Theorem A.8. Let $\mathscr{L}=(H, L, s, t, \gamma, \pi)$ be a left bialgebroid and $B$ an L-ring. Two weak crossed products of $B$ with $\mathscr{L}$ are equivalent if and only if they are related by a gauge transformation.

In order to make connection between weak crossed products and weak cleft extensions, the notion of invertible weak 2-cocycles is needed.

Definition A.9. Let $\mathscr{L}=(H, L, s, t, \gamma, \pi)$ be a left bialgebroid and $B$ an $L$-ring weakly measured by $\mathscr{L}$. An inverse of a $B$-valued weak 2 -cocycle $\sigma$ on $\mathscr{L}$ is a $k$-linear map, $\tilde{\sigma}: H \otimes_{L} H \rightarrow B$ (where the right and left $L$-module structures on $H$ are given by right and left multiplication by $s(l)$, respectively) satisfying properties (a), (b) and (c) in Definition 4.8 and, in addition, for all $h, k \in H$,

$$
\left(h_{(1)} k_{(1)} \cdot 1_{B}\right) \tilde{\sigma}\left(h_{(2)}, k_{(2)}\right)=\tilde{\sigma}(h, k) .
$$

If $\sigma$ is a 2-cocycle in the sense of Definition 4.2 (in particular the measuring satisfies also property (a) in Definition 4.1), then Definition A.9 is equivalent to Definition 4.8. By an argument similar to the proof of Lemma 4.9. the convolution inverse of a weak 2-cocycle is unique, provided it exists. A generalisation of Theorems 4.11 and 4.12 is given in the following

Theorem A.10. Let $\mathscr{H}$ be a Hopf algebroid. A right $\mathscr{H}$-extension $B \subseteq A$ is weak $\mathscr{H}$ cleft if and only if $A$ is isomorphic to a weak crossed product of $B$ with the constituent left bialgebroid $\mathscr{H}_{L}$ of $\mathscr{H}$, with respect to an invertible weak 2-cocycle.

Analogously to Corollary 4.13, Theorems A.10 and A.8 lead to the following

Corollary A.11. Let $\mathscr{H}$ be a Hopf algebroid and $B \subseteq A$ a weak $\mathscr{H}$-cleft extension. Let $j: H \rightarrow A$ be a weak cleaving map with left convolution inverse $j^{w}$, satisfying conditions (3.8) and (3.9). Then the map $h \cdot b:=j\left(h^{(1)}\right) b j^{w}\left(h^{(2)}\right)$, for all $b \in B$ and $h \in H$, is a weak measuring of the constituent left bialgebroid $\mathscr{H}_{L}$ on $B$. A map $j^{\prime}: H \rightarrow A$ is a weak cleaving map if and only if there exist morphisms $\chi, \tilde{\chi} \in \operatorname{Hom}_{L, L}(H, B)$, satisfying (A.2A.3), in terms of which $j^{\prime}: h \mapsto \chi\left(h_{(1)}\right) j\left(h_{(2)}\right)$.

Let $\mathscr{H}$ be a Hopf algebroid with a bijective antipode and let $B \subseteq A$ be a weak $\mathscr{H}$-cleft extension and $T$ a $k$-subalgebra of $B$. Let $j$ be a weak cleaving map with a left convolution inverse $j^{\mathrm{w}}$, satisfying conditions (3.8) and (3.9). Any morphism $f \in \operatorname{Hom}_{L, L}\left(H, B \otimes_{T} B\right)$ such that, for all $h \in H, \mu_{B}(f(h))=j\left(h^{(1)}\right) j^{\mathrm{w}}\left(h^{(2)}\right)$, determines a strong $T$-connection via (5.5). Conversely, any strong $T$-connection is of this form (though the correspondence (5.5) is not bijective in the weak case).

Any $B$ - $T$ bimodule section of a weak cleft Hopf algebroid extension $B \subseteq A$, for a subalgebra $T$ of $B$, corresponds to a left total $T$-integral via (5.6) (although the correspondence between $B-T$ sections and total integrals is not bijective in the weak case). Hence Corollary 5.9 is valid without modification for weak cleft extensions of Hopf algebroids with bijective antipode.

A weak Hopf algebra $(W, \Delta, \varepsilon, S)$ determines a Hopf algebroid $\mathscr{W}$ with constituent left bialgebroid $\mathscr{W}_{L}$ over the 'left' subalgebra $W^{L}$ of $W$, right bialgebroid $\mathscr{W}_{R}$ over the 'right' subalgebra $W^{R}$, and antipode $S$. The category of right comodules for the coalgebra $(W, \Delta, \varepsilon)$ is isomorphic to the category of right $\mathscr{W}$-comodules as a monoidal category. As a consequence, also the respective notions of comodule algebras and of coinvariants are equivalent 
(cf. [17]). Let $A$ be a right $W$ - (or, equivalently, $\mathscr{W}$-) comodule algebra with coinvariants $B$. By [3, Theorem 2.11], the extension $B \subseteq A$ is $W$-cleft (i.e. the corresponding weak entwining structure is cleft in the sense of [2, Definition 1.9]) if and only if it is $W$-Galois and $A$ is a direct summand of $B \otimes_{k} W$ as a left $B$ module right $W$-comodule. By [9, Example 3.5] the $W$-Galois property is equivalent to the $\mathscr{W}_{R}$-Galois property, hence Theorem A.2 implies that any weak $\mathscr{W}$-cleft extension is weak $W$-cleft (but not conversely).

\section{ACKNOWLEDGEMENTS}

GB is grateful to Kornél Szlachányi and Joost Vercruysse for inspiring discussions. Her work is supported by the Hungarian Scientific Research Fund OTKA T 043159 and the Bolyai János Fellowship.

\section{REFERENCES}

[1] J. Abuhlail, Morita contexts for corings and equivalences, [in:] Hopf algebras in noncommutative geometry and physics. S. Caenepeel and F. Van Oystaeyen (eds.), Lecture Notes in Pure and Appl. Math., 239, Marcel Dekker, New York, 2005, pp. 1-19.

[2] J.N. Alonso Álvarez, J.M. Fernández Vilaboa, R. González Rodríguez and A.B. Rodríguez Raposo, Weak C-cleft extensions, weak entwining structures and weak Hopf algebras, J. Algebra 284 (2005), 679-704.

[3] J.N. Alonso Álvarez, J.M. Fernández Vilaboa, R. González Rodríguez and A.B. Rodríguez Raposo, Weak C-cleft extensions and weak Galois extensions, J. Algebra 299 (2006), 276293.

[4] A. Ardizzoni, G. Böhm and C. Menini, A Schneider type theorem for Hopf algebroids, J. Algebra 318 (2007), 225-269. Corrigendum, to be published. See also http://arxiv.org/abs/math/0612633v2

[5] I. Bálint and K. Szlachányi, Finitary Galois extensions over non-commutative bases, J. Algebra 296 (2006), 520-560.

[6] R.J. Blattner, M. Cohen and S. Montgomery, Crossed products and inner actions of Hopf algebras, Trans. Amer. Math. Soc. 298 (1986), 671-711.

[7] R.J. Blattner and S. Montgomery, Crossed products and Galois extensions of Hopf algebras, Pacific J. Math. 137 (1989), 37-54.

[8] G. Böhm, Integral theory for Hopf algebroids, Alg. Rep. Theory 8(4) (2005), 563-599. Corrigendum, to be published. See also http://arxiv.org/abs/math/0403195, to be replaced by version 4 .

[9] G. Böhm, Galois theory for Hopf algebroids, Ann. Univ. Ferrara - Sez. VII - Sc. Mat. 51 (2005), 233-262. A corrected version is available at http://arxiv.org/abs/math/0409513v3

[10] G. Böhm, Hopfalgebroids, Preprint available at http://arxiv .org/abs/math/0805.3806 to appear in Handbook of Algebra Vol. 6, ed: M. Hazewinkel, Elsevier.

[11] G. Böhm and T. Brzeziński, Strong connections and the relative Chern-Galois character for corings, Int. Math. Res. Notices, 2005:42 (2005), 2579-2625.

[12] G. Böhm, F. Nill and K. Szlachányi, Weak Hopf algebras I: Integral theory and * $^{*}$-structure, J. Algebra 221 (1999), 385-438.

[13] G. Böhm and K. Szlachányi, Hopf algebroids with bijective antipodes: axioms, integrals and duals, J. Algebra 274 (2004) 708-750.

[14] G. Böhm and J. Vercruysse, Morita theory for coring extensions and cleft bicomodules, Adv. in Math. 209 (2007), 611-648. Corrigendum, to be published. See also http://arxiv.org/abs/math/0601464v2

[15] T. Brzeziński, On modules associated to coalgebra Galois extensions, J. Algebra 215 (1999), 290-317.

[16] T. Brzeziński, A note on coring extensions, Ann. Univ. Ferrara - Sez. VII - Sc. Mat. 51 (2005), 15-27. A corrected version is available at http://arxiv.org/abs/math/0410020v3. 
[17] T. Brzeziński, S. Caenepeel and G. Militaru, Doi-Koppinen modules for quantum groupoids, J. Pure Appl. Algebra 175 (2002), 45-62.

[18] T. Brzeziński and P.M. Hajac, Coalgebra extensions and algebra coextensions of Galois type, Comm. Alg. 27 (1999), 1347-1367.

[19] T. Brzeziński and S. Majid, Coalgebra bundles, Comm. Math. Phys. 191 (1998), 467-492.

[20] T. Brzeziński and R. Wisbauer, Corings and Comodules. Cambridge University Press, Cambridge, 2003. Erratum: http://www-maths.swan.ac.uk/staff/tb/corinerr.pdf

[21] S. Caenepeel and E. De Groot, Modules over weak entwining structures, AMS Contemp. Math. 267 (2000), 31-54.

[22] S. Caenepeel, J. Vercruysse and S. Wang, Morita theory for corings and cleft entwining structures, J. Algebra 276 (2004), 210-235.

[23] Y. Doi and M. Takeuchi, Cleft comodule algebras for a bialgebra, Comm. Algebra 14 (1986), 801-817.

[24] L. Kadison, Depth two and the Galois coring, in: Noncommutative geometry and representation theory in mathematical physics, 149-156, Contemp. Math., 391, Amer. Math. Soc., Providence, RI, 2005.

[25] L. Kadison, The Endomorphism Ring Theorem for Galois and D2 extensions, J. Algebra 305 (2006), 163-184.

[26] L. Kadison and K. Szlachányi, Bialgebroid actions on depth two extensions and duality, Adv. Math. 179 (2003), 75-121.

[27] J.-H. Lu, Hopf algebroids and quantum groupoids, Int. J. Math. 7 (1996) 47-70.

[28] P. Schauenburg, Bialgebras over noncommutative rings and a structure theorem for Hopf bimodules, Applied Categorical Structures 6 (1998), 193-222.

[29] M.E. Sweedler, Hopf Algebras. Benjamin, New York (1969).

[30] M. Takeuchi, Groups of algebras over $A \otimes \bar{A}$, J. Math. Soc. Japan 29 (1977) 459-492.

Research Institute for Particle and Nuclear Physics, Budapest, H-1525 BudAPEST 114, P.O.B. 49, Hungary

E-mail address: G.Bohm@rmki.kfki.hu

Department of Mathematics, University of Wales Swansea, Singleton Park, SWANSEA SA2 8PP, U.K.

E-mail address: T.Brzezinski@swansea.ac.uk 